\title{
IMPROVED LMI CONDITIONS FOR GAIN SCHEDULING AND RELATED CONTROL PROBLEMS
}

\author{
GÉRARD SCORLETTI ${ }^{1,2 *}$ AND LAURENT EL GHAOUI ${ }^{2}$ \\ ${ }^{1}$ Service Automatique, Ecole Supérieure d'Electricité, Plateau de Moulon, 91192 Gif-sur-Yvette, France \\ ${ }^{2}$ Laboratoire de Mathématiques Appliquées, Ecole Nationale Supérieure de Techniques Advancées, 32, Boulevard Victor, \\ 75739 Paris, France
}

\begin{abstract}
SUMMARY
The gain scheduling problem considered in this paper concerns a linear system whose state-space equations depend rationally on real, time-varying parameters, which are measured in real time. A stabilizing, parameter-dependent controller is sought, such that a given $\mathscr{L}_{2}$-gain bound for the closed-loop system is ensured. Sufficient linear matrix inequality (LMI) conditions are known, that guarantee the existence of such 'gain-scheduled' controllers.

This paper improves these results in two directions. First, we show how to exploit the realness of the parameters using a 'skew-symmetric scaling' technique. Moreover, we show how to apply this technique in a time-varying and/or nonlinear setting.

We first devise a general result pertaining to control synthesis of interconnection of dissipative operators, and apply it to the gain-scheduling problem. Owing to its generality, this result can be applied to other problems such as anti-windup control, nonlinear control and model reduction. (C) 1998 John Wiley \& Sons, Ltd.
\end{abstract}

Key words: gain scheduling; linear matrix inequality; anti-windup control

\section{INTRODUCTION}

\subsection{Problem definition}

We consider a parameter-dependent, continuous-time system:

$$
\begin{aligned}
\dot{x} & =\mathbf{A}(\delta(t)) x+\mathbf{B}_{w}(\delta(t)) w+\mathbf{B}_{u}(\delta(t)) u \\
z & =\mathbf{C}_{z}(\delta(t)) x+\mathbf{D}_{z w}(\delta(t)) w+\mathbf{D}_{z u}(\delta(t)) u \\
y & =\mathbf{C}_{y}(\delta(t)) x+\mathbf{D}_{y w}(\delta(t)) w+\mathbf{D}_{y u}(\delta(t)) u
\end{aligned}
$$

This paper was recommended for publication by editor A. Stoorvogel

\footnotetext{
* Correspondence to: G. Scorletti, Laboratoire de Mathematiques Appliquées, Ecole Nationale Superiéure de Techniques Avancées, 32, Boulevard Victor, 75739 Paris, France. E-mail: scorlett@ensta.fr
}

Contract/grant sponsor: MRES; Contact/grant number: 93-217

CCC 1049-8923/98/100845-33\$17.50

(C) 1998 John Wiley \& Sons, Ltd. 
where $x \in \mathbf{R}^{n}$ is the state vector, $u \in \mathbf{R}^{n_{u}}$ is the command input, $y \in \mathbf{R}^{n_{y}}$ is the measured output, $z \in \mathbf{R}^{n_{z}}$ is the output of interest and $w \in \mathbf{R}^{n_{w}}$ is the disturbance input. The vector $\delta(t) \in \mathbf{R}^{r}$ is a (time-varying, real) parameter vector. The state-space matrices $\mathbf{A}, \mathbf{B}_{w}, \mathbf{B}_{u}, \mathbf{C}_{z}, \mathbf{D}_{z w}, \mathbf{D}_{z u}, \mathbf{C}_{y}, \mathbf{D}_{y w}$ and $\mathbf{D}_{y u}$ are well-defined (multivariable) rational functions of $\delta(t)$.

We assume that the parameter vector $\delta(t)$ is measured in real time, and is known a priori to belong to given intervals: $\delta_{i}(t) \in\left[a_{i}, b_{i}\right]$ for every $t \geqslant 0$ and $i=1, \ldots, r$. Without loss of generality, we assume that every interval $\left[a_{i}, b_{i}\right]$ contains 0 .

Our main problem is to derive sufficient conditions for the existence of a parameter-dependent output-feedback controller that ensures, for the closed-loop system, (i) stability (ii) a given $\mathscr{L}_{2}$-gain bound from $w$ to $z$. In the sequel, this problem is referred to as the "nominal gainscheduling problem'. It is the main prblem considered in this paper, but we consider several related problems that are described in more detail in Section 1.4.

\subsection{Related work}

Gain-scheduled control has been for decades a well-known engineering practice, with little theoretical justification. A breakthrough occurred in 1991 with the papers of Doyle, Packard, Zhou, Lu, Pandey and Becker. ${ }^{1-3}$ Using the 'linear fractional transformation' (LFT) representation for the system, conditions for the eistence of a parameter-dependent controller that guarantees stability and $\mathbf{H}_{\infty}$ performance for the closed-loop system are given in the form of linear matrix inequalities (LMIs). These conditions are based on a scaled version of the small-gain theorem, with a symmetric scaling matrix.

In Reference 4, Packard considers the gain-scheduling problem for linear systems with rational parameter dependence. LFT representations and LMI tools are used to obtain sufficient conditions for the controller synthesis problem. As the author notes, this approach does not exploit the realness of the parameters. Packard only treated dicrete-time systems. The case of continuoustime systems was dealt with by Apkarian and Gahinet. ${ }^{5}$

As Packard notes, ${ }^{4}$ parameter realness can be taken into account using the approach proposed by Becker and co-authors. ${ }^{6-9}$ Becker considers the control of parameter-dependent systems, assuming that the state-space matrices depend linearly on the parameters taken in a polytope. He obtains an LMI formulation, the size of which grows exponentially with the number of parameters.

Improved LMI conditions with reasonable size were proposed by Helmersson. ${ }^{10,11}$ Helmersson devised an algebraic framework, in which the system is represented as the linear fractional transformation of a matrix $M$ and a (complex matrix) $\Delta$, with maximum singular value or $\mathscr{L}_{2}$ norm less than one. (This is the familiar framework for robustness analysis of LTI systems.) The obtained conditions are similar to ours. However, the proposed framework is not adapted to time-varying systems, since a formal analogy is made with the $\mu$-analysis problem for LTI systems.

An alternative approach to the gain-scheduling problem was recently proposed by $\mathrm{Wu}$ and co-authors. ${ }^{12}$ Instead of using the scaled small-gain theorem, Wu considers a parameter-dependent Lyapunov function. This approach was first proposed for the analysis of gain-scheduled control by Fromion et al. ${ }^{13}$ The main motivation here is to take into account a priori bounds on the rate of variation of the parameters. Unfortunately, contrary to previous approaches to the gain-scheduling problem, ${ }^{4,5}$ the resulting conditions can be checked only approximately, with a high computational burden. More precisely, Wu's conditions require a gridding on the set of the 
parameters in conjunction with the resolution of a high number of LMI problems. This approach has one of the drawbacks of the traditional gain-scheduling engineering practice: there is no guarantee on the stability of the closed-loop system.

\subsection{Main contribution}

In this paper, we obtain new (sufficient) LMI conditions for the gain-scheduling problem. Our conditions improve those in Reference 4 in that they take into account explicitly the realness of the parameters, via a skew-symmetric scaling technique. In addition, these conditions remain as numerically attractive as Packard's.

To derive our results, we consider a general control problem for interconnected systems, which can be represented as the connection of a block-diagonal operator $\Delta$ and a constant matrix $M$, called the interconnection matrix. The block-diagonal operator $\Delta$ satisfies a dissipative property, expressed in the form of a time-domain integral quadratic constraint on its inputs and outputs. We obtain synthesis conditions for the existence of a controller that (i) stabilizes the interconnected system (ii) ensures a dissipative property between a given input-output pair.

The study of (nonlinear, time-varying, etc.) interconnected systems started in the 60-70s, motivated by large-scale systems and network analysis. ${ }^{14,15}$ This paper extends these results to a number of synthesis problems, in a nonlinear, time-varying setting. Our approach is closely related to those taken in References 16-18 for the analysis of systems.

\subsection{Related problems}

Our main result can in fact be applied to a variety of control problems involving interconnection of operators. In addition to the nominal gain-scheduling problem, five other related problems are considered.

- The robust gain-scheduling problem is an extension of the nominal problem to the case when (i) the scheduling parameters are measured with a (deterministic) bounded error, (ii) (nonlinear, dynamic) uncertainties perturb the (nonlinear) dynamics of the system.

- The anti-windup control problem is that of controlling linear time-invariant (LTI) systems with input saturations. This problem is the subject of intense research, as saturations are almost always present at the input of the plant. A now classical approach is the anti wind-up design: ${ }^{19-21}$ a controller is designed ignoring the saturations; this control law is modified to compensate for the effects of the nonlinearities; the stability of the closed-loop system is then checked. It is thus a 'trial and error' approach. We propose an approach which directly gives an anti-windup controller, without iteration. This philosophy was adopted in Reference 22 to obtain a more specific result, pertaining to positive real systems.

- The model reduction problem deals with model reduction for uncertain systems. An uncertain system can be modelled as the interconnection of an LTI system and a linear (dissipative) operator. For a given system, such interconnection models are not unique. To obtain a tractable problem (for robustness analysis or for control system synthesis), a model where the 'size of the uncertainty' is as small as possible is desirable. This problem is a generalization of the $H_{\infty}$ model reduction of LTI systems (see e.g. Reference 23). A numerical approach to the model-reduction problem was first considered by Beck and co-authors, ${ }^{24-26}$ leading to a non-convex formulation, whose efficient resolution is still an open problem. 
- The control of nonlinear rational systems problem concerns a (very general) class of nonlinear systems considered in Reference 27. A methodology based on convex optimization was proposed there to achieve quadratic stabilization. Here again, we provide improved conditions for this problem.

- The control of static systems problem concerns a class of matrix problems (systems with no dynamics). Their possible applications are detailed in Section 4.6.

\subsection{Paper outline}

The paper is organized as follows. In Section 2, the interconnected systems under consideration are described and the control problem under consideration is briefly presented. The main result, that is, control conditions for interconnected systems, is provided in Section 3. Section 4 addresses the application of the obtained conditions to the nominal/robust gain-scheduling control, anti-windup control, control of nonlinear rational systems, model reduction and control of static systems. All the proofs are given in the Appendix. This paper describes a generalization of the results given in the conference papers. ${ }^{28,29}$

The nominal gain-scheduling problem is chosen as a step-by-step illustration throughout the theoretical part.

Notations. $\mathscr{L}_{2}$ denotes the set of square integrable signals. For an $\mathscr{L}_{2}$-integrable signal $w,\|w\|$ denotes its $\mathscr{L}_{2}$ norm. For a signal $w$ and a given $T$, the causal truncation $P_{T}(x)$ at time $T$ is defined by

$$
\begin{array}{ll}
P_{T}(x(t))=x(t) & t \leqslant T \\
P_{T}(x(t))=0 & t>T
\end{array}
$$

$\mathscr{L}_{2 e}$ denotes the set of signals whose truncation is square integrable for any $T$. $\|w\|_{T}$ denotes the $\mathscr{L}_{2}$-norm of the truncation at time $T$ of the signal $w$.

$I_{r}$ and $0_{r}$ denote the identity and the zero matrices of $\mathbf{R}^{r \times r}$, with $I_{0}$ (or $0_{0}$ ) the empty matix. The subscript is omitted when obvious. For a matrix $X, X^{k}$ denotes the upper left $k \times k$ submatrix. For two operators $A$ and $B, \operatorname{diag}(A, B)$ denotes the operator $\left[\begin{array}{cc}A & 0 \\ 0 & B\end{array}\right]$. We denote by $\mathscr{P}_{\mathcal{M}}$ and $\mathscr{P}_{\mathcal{N}}$ two permutation matrices associated with the integers $n, n_{w}$ and $n_{z}$ :

$$
\mathscr{P}_{\mathscr{M}}=\left[\begin{array}{cccc}
I_{n} & 0 & 0 & 0 \\
0 & 0 & I_{n} & 0 \\
0 & I_{n_{z}} & 0 & 0 \\
0 & 0 & 0 & I_{n_{w}}
\end{array}\right] \mathscr{P}_{\mathscr{N}}=\left[\begin{array}{cccc}
I_{n} & 0 & 0 & 0 \\
0 & 0 & I_{n} & 0 \\
0 & I_{n_{w}} & 0 & 0 \\
0 & 0 & 0 & I_{n_{z}}
\end{array}\right]
$$

Let $U$ be a full-rank $r \times n$ real matrix with $r<n$. $U^{\perp}$ denotes an orthogonal complement of $U$, i.e., $U U^{\perp}=0$ and $\left[U^{\mathrm{T}} U^{\perp}\right]$ is of maximal rank. $U^{+}$is the Moore-Penrose inverse of $U$. $\lambda_{\max }(A)$ denotes the eigenvalue with the largest real part of the matrix $A$. For a given integer $n$, we define the sets

$$
\mathscr{S}(n)=\left\{S \in \mathbf{R}^{n \times n}, S=S^{\mathrm{T}}>0\right\} \quad \text { and } \quad \mathscr{G}(n)=\left\{G \in \mathbf{R}^{n \times n}, G=-G^{\mathrm{T}}\right\}
$$

If $M$ is a matrix, partitioned as $M=\left[\begin{array}{ll}M_{11} & M_{12} \\ M_{21} & M_{22}\end{array}\right]$ and $\Delta$ is an operator then the notation $\mathscr{F}_{u}(M, \Delta)$ stands for

$$
\mathscr{F}_{u}(M, \Delta) \triangleq M_{22}+M_{21} \Delta\left(I-M_{11} \Delta\right)^{-1} M_{12}
$$


Table I. Classes of operators $\Delta$

\begin{tabular}{lcccccc}
\hline Class & $\mathscr{C}_{1}$ & $\mathscr{C}_{2}$ & $\mathscr{C}_{3}$ & $\mathscr{C}_{4}$ & $\mathscr{C}_{5}$ & $\mathscr{C}_{6}$ \\
\hline Structure & $g_{i} I_{n_{i}}$ & $\mathscr{G}_{i}$ & $\delta_{i}(t) I_{n_{i}}$ & $\Delta_{i}(t)$ & $\Delta_{n i}(\cdot)$ & $\Delta_{m i}(\cdot)$ \\
\hline Type & $\begin{array}{l}g_{i} \text { SISO } \\
\text { linear }\end{array}$ & $\begin{array}{l}\text { MIMO } \\
\text { linear }\end{array}$ & $\begin{array}{l}\delta_{i}(t) \text { time-varying } \\
\text { real scalar }\end{array}$ & $\begin{array}{l}\text { time-varying } \\
\text { real matrix }\end{array}$ & $\begin{array}{l}\text { MIMO } \\
\text { nonlinear }\end{array}$ & $\begin{array}{l}\text { MIMO } \\
\text { memory } \\
\text { less }\end{array}$ \\
\hline
\end{tabular}

whenever it is well posed. MIMO stands for multi-inputs multi-outputs, SISO for single-input and single-output, and LTI for linear time invariant.

\section{PROBLEM FORMULATION}

\subsection{Interconnected systems}

Definition. In this paper, we define an interconnected system as one of the form

$$
\left[\begin{array}{c}
q(t) \\
z(t) \\
\hline y(t)
\end{array}\right]=\left[\begin{array}{c|c}
M & M_{u} \\
\hline M_{y} & 0
\end{array}\right]\left[\begin{array}{c}
p(t) \\
\frac{w(t)}{u(t)}
\end{array}\right] \text { and } p(t)=\Delta(q(t))
$$

where $M$ is a constant matrix, and $\Delta$ is a (causal) operator from $\mathscr{L}_{2 e}$ to $\mathscr{L}_{2 e}$, with dimensions smaller than those of $M$. The signal $z(t) \in \mathbf{R}^{n_{z}}$ is the output of the system, $y(t) \in \mathbf{R}^{n_{y}}$ is the measured output, $w(t) \in \mathbf{R}^{n_{w}}$ is the disturbance input and $u(t) \in \mathbf{R}^{n_{u}}$ is the command input. The signals $p(t)$ and $q(t)$ are internal variables. In the sequel, the interconnected system is written as

$$
\left[\begin{array}{l}
z(t) \\
y(t)
\end{array}\right]=\mathscr{F}_{u}\left(\left[\begin{array}{cc}
M & M_{u} \\
M_{y} & 0
\end{array}\right], \Delta\right)\left[\begin{array}{l}
w(t) \\
u(t)
\end{array}\right]
$$

whenever the above expression is well posed.

Let us now define which classes of operators $\Delta$ we consider. We assume that $\Delta$ is block diagonal: $\Delta=\operatorname{diag}\left(\Delta_{1}, \ldots, \Delta_{r}\right)$, where each $\Delta_{i}$ is square, without loss of generality. Each block - which can be thought of as a subsystem - is assumed to belong to one of the classes $\mathscr{C}_{1}, \ldots, \mathscr{C}_{6}$ that are listed in Table I.

We make a few comments on the terminology used in Table I. The words 'linear systems' refer to linear time-varying operators described by equations of the form:

$$
\begin{aligned}
\dot{x}_{\Delta_{i}} & =A(t) x_{\Delta_{i}}+B(t) q_{i} \\
p_{i} & =C(t) x_{\Delta_{i}}+D(t) q_{i}
\end{aligned}
$$

where $A(t), B(t), C(t)$ and $D(t)$ are bounded, piecewise continuous functions. The symbol $\delta I_{n_{i}}$ is just a notation for the operator $\operatorname{diag}(\delta, \ldots, \delta)$ : in this case, the operator is called a 'repeated' block. Otherwise, it is a 'full' block. 
The class $\mathscr{C}_{5}$ of MIMO nonlinear operators refers to the class of nonlinear operators that are described by ordinary differential equations:

$$
\begin{aligned}
\dot{x}_{\Delta_{i}} & =f\left(x_{\Delta_{i}}, q_{i}, t\right) \\
p_{i} & =h\left(x_{\Delta_{i}}, t\right)
\end{aligned}
$$

with $f$ and $h$ Lipschitz continuous. (This kind of operator is strongly causal, see Reference 30; pp. 14,99 ; the strong form of causality is important to ensure well-posedness of the interconnected system.)

Finally, the class $\mathscr{C}_{6}$ of MIMO memoryless operators refers to (time-dependent) nonlinear functions, that is, relations of the form $p_{i}=\Delta_{i}\left(q_{i}, t\right)$.

Example. The parameter-dependent system (1) is an example of an interconnected system. As shown in e.g. References 31, every rational matrix-valued function can be represented in a 'linearfractional transformation' (LFT) form. That is, given the rational functions $\mathbf{A}, \mathbf{B}_{u}$, etc. there exist matrices $A, B_{u}$, etc., such that, whenever the expressions are well defined, we have

$$
\begin{aligned}
\mathbf{M}(\delta) & =\left[\begin{array}{ccc}
\mathbf{A}(\delta) & \mathbf{B}_{w}(\delta) & \mathbf{B}_{u}(\delta) \\
\mathbf{C}_{z}(\delta) & \mathbf{D}_{z w}(\delta) & \mathbf{D}_{z u}(\delta) \\
\mathbf{C}_{y}(\delta) & \mathbf{D}_{y w}(\delta) & \mathbf{D}_{y u}(\delta)
\end{array}\right] \\
& =\left[\begin{array}{ccc}
A & B_{w} & B_{u} \\
C_{z} & D_{z w} & D_{z u} \\
C_{y} & D_{y w} & D_{y u}
\end{array}\right]+\left[\begin{array}{c}
B_{p} \\
D_{z p} \\
D_{y p}
\end{array}\right] \Delta_{\mathrm{par}}(t)\left(I-D_{q p} \Delta_{\mathrm{par}}(t)\right)^{-1}\left[\begin{array}{lll}
C_{q} & D_{q w} & D_{q u}
\end{array}\right]
\end{aligned}
$$

where

$$
\Delta_{\mathrm{par}}(t)=\operatorname{diag}\left(\delta_{1}(t) I_{n_{1}}, \ldots, \delta_{r}(t) I_{n_{r}}\right)
$$

This shows that system (1) can be represented as (2), with

$$
\left[\begin{array}{c|c}
M & M_{u} \\
\hline M_{y} & 0
\end{array}\right]=\left[\begin{array}{ccc|c}
A & B_{p} & B_{w} & B_{u} \\
C_{q} & D_{q p} & D_{q w} & D_{q u} \\
C_{z} & D_{z p} & D_{z w} & D_{z u} \\
\hline C_{y} & D_{y p} & D_{y w} & D_{y u}
\end{array}\right]
$$

and

$$
\Delta=\operatorname{diag}\left(\int I_{n}, \delta_{1}(t) I_{n_{1}}, \ldots, \delta_{r}(t) I_{n_{r}}\right)
$$

where $\int$ is the integration operator. With the terminology listed in Table I, the first block of $\Delta$ belongs to class $\mathscr{C}_{1}$, and the other blocks to class $\mathscr{C}_{3}$.

\subsection{Interconnected systems with integral quadratic constraints}

Motivation and definition. In this paper, we assume that the operator $\Delta$ satisfies a known integral quadratic constraint, for which we give a definition below. 
Table II. Structure of the integral quadratic constraints that are considered for the classes of operators $\Delta_{i}$ defined in Table I

\begin{tabular}{lcc}
\hline Class & $\mathscr{C}_{1}$ and $\mathscr{C}_{3}$ & $\mathscr{C}_{2}, \mathscr{C}_{4}, \mathscr{C}_{5}, \mathscr{C}_{6}$ \\
\hline$\Phi_{i}$ & {$\left[\begin{array}{ll}x_{i} I_{n_{i}} & y_{i} I_{n_{i}} \\
y_{i} I_{n_{1}} & z_{i} I_{n_{1}}\end{array}\right], x_{i}, y_{i}, z_{i}$ scalars } & {$\left[\begin{array}{cc}X_{i} & Y_{i} \\
Y_{i}^{T} & Z_{i}\end{array}\right], X_{i}, Y_{i}, Z_{i} \in \mathbf{R}_{n_{i} \times n_{i}}$} \\
\hline
\end{tabular}

\section{Definition 2.1}

Let $X, Y$ and $Z$ be (constant, real) matrices such that $X>0$ or $X=0, Z \leqslant 0$ and let the full rank matrix

$$
\Phi=\left[\begin{array}{ll}
X & Y \\
Y^{\mathrm{T}} & Z
\end{array}\right]
$$

Let $H: \mathscr{L}_{2 e} \rightarrow \mathscr{L}_{2 e}$ be an operator. We say that $H$ is $\{X, Y, Z\}$-dissipative if for every $p, q \in \mathscr{L}_{2 e}, p=H(q)$ implies that:

$$
\text { for every } T \geqslant 0, \int_{0}^{T}\left[\begin{array}{l}
q(t) \\
p(t)
\end{array}\right]^{\mathrm{T}} \Phi\left[\begin{array}{l}
q(t) \\
p(t)
\end{array}\right] \mathrm{d} t \geqslant 0
$$

In the sequel, the condition (6) is referred as an integral quadratic constraint.

Note that if $X=I, Y=0$ and $Z=-I$, then the property above expresses that the operator $H$ has an $\mathscr{L}_{2}$-gain less than one. The choice $X=0, Y=I$ and $Z=0$ expresses that the operator $H$ is passive. Note also that we can write integral quadratic constraints for block-diagonal operators, using block-diagonal matrices $\Phi$. Precisely, if the operators $\Delta_{i}$ are $\left\{X_{i}, Y_{i}, Z_{i}\right\}$-dissipative then the operator $\Delta=\operatorname{diag}\left(\Delta_{1}, \ldots, \Delta_{r}\right)$ is $\{X, Y, Z\}$-dissipative with $X=\operatorname{diag}$ $\left(X_{1}, \ldots, X_{r}\right), Y=\operatorname{diag}\left(Y_{1}, \ldots, Y_{r}\right)$ and $Z=\operatorname{diag}\left(Z_{1}, \ldots, Z_{r}\right)$.

In Table II, we list the structure of the integral quadratic constraints, that we are allowed to consider in this paper.

In the sequel, we denote by $\Delta$ the set of admissible operators $\Delta$. This set is defined via the classes $\mathscr{C}_{1}, \ldots, \mathscr{C}_{6}$ to which every diagonal block of $\Delta$ belongs, and also by an integral quadratic constraint that $\Delta$ is known to satisfy.

Example. To take an example, return to the parameter-dependent system described by (1). Recall that this kind of system can be written as (2), with $\Delta$ given in (5). The first block of $\Delta$ is an integration operator, therefore it satisfies an integral quadratic constraint of the form (6), with $\Phi$ replaced by $\Phi_{0}$, where

$$
\Phi_{0}=\left[\begin{array}{cc}
X_{0} & Y_{0} \\
Y_{0}^{\mathrm{T}} & Z_{0}
\end{array}\right]=\left[\begin{array}{ll}
0 & I \\
I & 0
\end{array}\right]
$$

If we assume that each parameter $\delta_{i}$ is known to lie within given intervals $\left[a_{i}, b_{i}\right]$, then the operator block $\Delta_{i}(t)=\delta_{i}(t) I_{n_{i}}$ satisfies an integral quadratic constraint of the form (6), where $\Phi$ is replaced by

$$
\Phi_{i}=\left[\begin{array}{cc}
X_{i} & Y_{i} \\
Y_{i}^{\mathrm{T}} & Z_{i}
\end{array}\right]=\left[\begin{array}{cc}
-2 a_{i} b_{i} I & \left(a_{i}+b_{i}\right) I \\
\left(a_{i}+b_{i}\right) I & -2 I
\end{array}\right]
$$


The overall operator $\Delta$ satisfies an integral quadratic constraint with

$$
X=\operatorname{diag}\left(X_{0}, X_{1}, \ldots, X_{r}\right), Y=\operatorname{diag}\left(Y_{0}, Y_{1}, \ldots, Y_{r}\right) \text { and } Z=\operatorname{diag}\left(Z_{0}, Z_{1}, \ldots, Z_{r}\right)
$$

Comments. We make a few comments on our definition of integral quadratic constraints, which is similar in nature to those introduced in References 16 and 32, in the context of large-scale system stability analysis.

Our definition is different from the integral quadratic constraints (IQC) proposed by Rantzer and Megretsky. ${ }^{18}$ In Definition 2.1, the matrix $\Phi$ is restricted to be constant, and $X$ and $Z$ must be semidefinite - we are thus more restrictive in our definition than in the context of Reference 18. However, we allow some (input/output) unstable operators $H$, which is not allowed in the IQC framework. Note that if $H$ is an integrator, then

$$
\text { for every } T \geqslant 0, \quad \int_{0}^{T}\left[\begin{array}{l}
q(t) \\
p(t)
\end{array}\right]^{\mathrm{T}}\left[\begin{array}{ll}
0 & I \\
I & 0
\end{array}\right]\left[\begin{array}{l}
q(t) \\
p(t)
\end{array}\right] \mathrm{d} t \geqslant 0
$$

An integrator does not satisfy an IQC, as defined by Reference 18: it is not input/output stable.

The restrictions imposed on the matrix $\Phi$ that defines the integral quadratic constraint serve several other purposes. The condition $Z \leqslant 0$ implies that:

- the set of operators $H$ which satisfy (6) is convex (an important fact needed in our proofs),

- when $H$ is stable, we recover a (special kind of) IQC. Precisely, the condition

$$
\int_{0}^{+\infty}\left[\begin{array}{c}
q(t) \\
p(t)
\end{array}\right]^{\mathrm{T}} \Phi\left[\begin{array}{c}
q(t) \\
p(t)
\end{array}\right] \mathrm{d} t \geqslant 0
$$

is equivalent to condition (6).

The condition $X \geqslant 0$ ensures that the set of operators $H$ which satisfy (6) contains the operator 0

\subsection{Robust gain-scheduled control problem}

Controller structure. For the interconnected system defined in Section 2.1, we will seek a controller which achieves specifications given later, with a structure linked to that of the operator $\Delta$. The admissible controller structures are given below.

\section{Definition 2.2}

A robust gain-scheduled controller is an interconnected system of the form $u=\mathscr{F}_{u}\left(K, \Delta_{K}\right) y$, where $K$ is a constant matrix, and

where

$$
\Delta_{K}=\operatorname{diag}\left(\Delta_{K 1}, \ldots, \Delta_{K r}\right)
$$

- if $\Delta_{i}$ is a repeated linear block (classes $\mathscr{C}_{1}$ and $\mathscr{C}_{3}$ ), that is, $\Delta_{i}=\delta_{i} I_{n_{i}}$ then $\Delta_{K i}=\delta_{i} I_{k i}$ where $k_{i}$ is a given integer $k_{i} \leqslant n_{i}$;

- if $\Delta_{i}$ is a linear full block (classes $\mathscr{C}_{2}$ and $\mathscr{C}_{4}$ ), then $\Delta_{K i}=\Delta_{i}$ or $\Delta_{K i}=I_{0}$;

- if $\Delta_{i}$ is a nonlinear full block (classes $\mathscr{C}_{5}$ and $\mathscr{C}_{6}$ ), then $\Delta_{K_{i}}=I_{0}$.

Two cases of robust gain-scheduled controllers are of special interest.

- The fully robust controller is one of the form $u=K y$, where $K$ is a constant matrix. 
- The gain-scheduled controller corresponds to the case when $\Delta$ contains no nonlinear elements, and $\Delta_{K}=\Delta$, that is, the control law is of the form $u=\mathscr{F}_{u}(K, \Delta) y$, where $K$ is a constant matrix.

In the sequel, if $\Delta_{K}=\Delta$ then the controller is said to contain a full copy of $\Delta$. Otherwise, it is said to contain a partial copy of $\Delta$. We note that the structure of the controller is determined by that of the matrix $\Delta_{K}$, and results from a choice of the user. Once this structure is chosen, the control problem, detailed below, will be to find an appropriate matrix $K$.

Problem definition. To define our control problem, we first recall the standard notion of (internal) stability for interconnected systems.

\section{Definition 2.3}

The system

$$
\left[\begin{array}{l}
q(t) \\
z(t)
\end{array}\right]=M\left[\begin{array}{l}
p(t) \\
w(t)
\end{array}\right] \text { and } \quad p(t)=\Delta(q)(t)
$$

is said to be (internally) $\mathscr{L}_{2}$-gain stable if for any $w$, there exist a $k>0$ and unique $p, q, z$, which depend causally on $w$, such that:

$$
\forall w \in \mathscr{L}_{2},\|p\| \leqslant k\|w\|, \quad\|q\| \leqslant k\|w\| \quad \text { and } \quad\|z\| \leqslant k\|w\|
$$

Our main problem is as follows.

\section{General control problem}

- Given an interconnected system (1), where each block of $\Delta$ belongs to a given class, and satisfies a given integral quadratic constraint (as listed in Table 2),

- given a controller structure (that is, an operator $\Delta_{K}$, as defined in Definition 2.2),

find a 'robust gain-scheduled controller' (that is, a constant matrix $K$, see Definition 2.2), which achieves for the closed-loop system (see Figure 1) (i) stability (ii) an $\left\{X_{\text {perf }}, Y_{\text {perf }}, Z_{\text {perf }}\right\}$-dissipativity property between the input $w$ and the output $z$.

Example. To illustrate the above, we return to the nominal gain-scheduling control problem referred to in Section 1.1. In this problem, we start from an interconnected system, as defined by (4) and (5). In view of the bounds on the parameters $\delta_{i}$, this system satisfies an $\{X, Y, Z\}$ dissipativity property, with $X, Y, Z$ defined by (7). We seek to ensure stability and a given $\mathscr{L}_{2}$-gain performance bound $\gamma$ (for the map from $w$ to $z$ ). This corresponds to the above control problem, with $X_{\text {perf }}=\gamma^{2} I, Y_{\text {perf }}=0$ and $Z_{\text {perf }}=-I$.

The controller structure is as follows. Since the parameters are measured, they are available in real time to the controller. Moreover, the controller is of the same order as that of the plant; therefore, it contains as many integrators as the open-loop system. The controller structure is thus chosen to be 'gain-scheduled', that is, $\Delta_{K}=\Delta$ (the controller contains a full copy of $\Delta$ ). The 


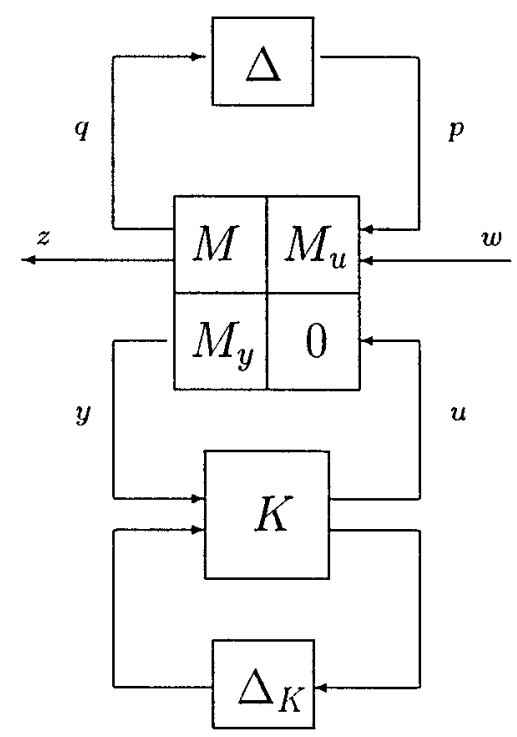

Figure 1. Robust gain-scheduled control

controller can therefore be written as

$$
\begin{gathered}
\dot{x}_{K}=\mathbf{A}_{K}(\delta(t)) x_{K}+\mathbf{B}_{K}(\delta(t)) y \\
u=\mathbf{C}_{K}(\delta(t)) x_{K}+\mathbf{D}_{K}(\delta(t)) y
\end{gathered}
$$

where $x_{K} \in \mathbf{R}^{n}$, and

$$
\left[\begin{array}{ll}
\mathbf{A}_{K}(\delta) & \mathbf{B}_{K}(\delta) \\
\mathbf{C}_{K}(\delta) & \mathbf{D}_{K}(\delta)
\end{array}\right]=\mathrm{F}_{u}\left(K, \Delta_{\mathrm{par}}(t)\right), \quad \Delta_{\mathrm{par}}(t)=\operatorname{diag}\left(\delta_{1}(t) I_{n_{1}}, \ldots, \delta_{r}(t) I_{n_{r}}\right)
$$

\section{MAIN RESULTS}

\subsection{Scalings}

To analyse interconnected systems of the class defined before is usually very difficult. We must take into account two facts: first, the information on the class of $\Delta$ (full, repeated, real, etc.); second, the information that the operator $\Delta$ satisfies a given integral quadratic constraint.

One approach, taken in this paper, is to replace the above two pieces of information by a family of integral quadratic constraints that are parametrized by 'scaling' matrices. To this end, we introduce the sets $\underline{\mathbf{S}}(\boldsymbol{\Delta}), \underline{\mathbf{G}}(\boldsymbol{\Delta})$, defined by

$$
\begin{aligned}
& \underline{\mathbf{S}}(\boldsymbol{\Delta})=\left\{\mathbf{S} \mid S^{\mathrm{T}}=S^{\mathrm{T}}>0, \text { for every } \Delta \in \Delta, S^{1 / 2} \Delta S^{-1 / 2} \in \Delta\right\} \\
& \underline{\mathbf{G}}(\boldsymbol{\Delta})=\left\{G \mid \forall \Delta \in \Delta, \forall q, \Delta(q)^{\mathrm{T}} G q+(G q)^{\mathrm{T}} \Delta(q)=0\right\}
\end{aligned}
$$

Note that $\underline{\mathbf{S}}(\boldsymbol{\Delta}), \underline{\mathbf{G}}(\boldsymbol{\Delta})$ are both linear sets. 
Table III. Scalings $S_{i}$ and $G_{i}$

\begin{tabular}{lcll}
\hline Class & $\mathscr{C}_{1}$ & $\mathscr{C}_{3}$ & $\mathscr{C}_{2}, \mathscr{C}_{4}, \mathscr{C}_{5}$ and $\mathscr{C}_{6}$ \\
\hline$S_{i}$ & $S_{i} \in \mathscr{S}\left(n_{i}\right)$ & $S_{i} \in \mathscr{S}\left(n_{i}\right)$ & $S_{i}=\lambda_{i} I_{n_{i}}$ and $\lambda_{i}>0$ \\
$G_{i}$ & $G_{i}=0$ & $G_{i} \in \mathscr{G}\left(n_{i}\right)$ & $G_{i}=0$ \\
\hline
\end{tabular}

\section{Lemma 3.1}

Consider the operator $\Delta$ introduced in Section 2. If an operator $\Delta$ is $\{X, Y, Z\}$-dissipative, then for every $S \in \underline{\mathbf{S}}(\Delta), G \in \underline{\mathbf{G}}(\Delta)$, the operator $\Delta$ is also $\{X S, Y S+G, Z S\}$-dissipative.

It is obvious that the scaling matrices $S, G$ inherit the same block-diagonal structure as that of $\Delta: S=\operatorname{diag}\left(S_{1}, \ldots, S_{r}\right), G=\operatorname{diag}\left(G_{1}, \ldots, G_{r}\right)$, with $S_{i}, G_{i}$ of the same size as $\Delta_{i}$. In Table III, we have detailed the structure of the block-diagonal scalings $S_{i}, G_{i}$, depending on the class of the block $\Delta_{i}$.

Lemma 3.1 shows how to replace the two pieces of information on class and integral quadratic constraint, by a family of integral quadratic constraints that are parametrized by scaling matrices.

Example. To take an example, return to the parameter-dependent system described by (1). The first block of $\Delta$ is an integration operator, therefore it satisfies a family of integral quadratic constraints of the form (6) where $\Phi=\Phi_{0}(P)$ is parametrized by the matrix $P \in \mathscr{S}(n)$ :

$$
\text { for every } P, \quad \Phi_{0}(P)=\left[\begin{array}{ll}
0 & P \\
P & 0
\end{array}\right]
$$

Every block $\delta_{i}(t) I_{n_{i}}$ satisfies a family of integral quadratic constraints of the form (6), where $\Phi$ is parametrized by the matrices $S_{i} \in \mathscr{S}(n)$ and $G_{i} \in \mathscr{G}\left(n_{i}\right)$ :

$$
\Phi_{i}\left(S_{i}, G_{i}\right)=\left[\begin{array}{cc}
-2 a_{i} b_{i} S_{i} & \left(a_{i}+b_{i}\right) S_{i}+G_{i} \\
\left(a_{i}+b_{i}\right) S_{i}+G_{i}^{\mathrm{T}} & -2 S_{i}
\end{array}\right]
$$

The overall $\Delta$ satisfies a family of integral quadratic constraints with

$$
\begin{aligned}
& X=\operatorname{diag}\left(0,-2 a_{1} b_{1} S_{1}, \ldots,-2 a_{r} b_{r} S_{r}\right) \\
& Y=\operatorname{diag}\left(P,\left(a_{1}+b_{1}\right) S_{1}+G_{1}, \ldots,\left(a_{r}+b_{r}\right) S_{r}+G_{r}\right) \\
& Z=\operatorname{diag}\left(0,-2 S_{1}, \ldots,-2 S_{r}\right)
\end{aligned}
$$

\subsection{Analysis result}

We now give a sufficient condition ensuring that the interconnected system $z=\mathscr{F}_{u}(M, \Delta) w$ is stable and dissipative. The following theorem can be interpreted from a graph separation point of view. ${ }^{17,33}$ 


\section{Lemma 3.2}

The interconnected system $z=\mathscr{F}_{u}(M, \Delta) w$ is stable and $\left\{X_{\text {perf }}, Y_{\text {perf }}, Z_{\text {perf }}\right\}$-dissipative if there exist $S \in \underline{\mathbf{S}}(\boldsymbol{\Delta})$ and $G \in \underline{\mathbf{G}}(\boldsymbol{\Delta})$, such that

$$
\left[\begin{array}{c}
M \\
I_{\left(n+n_{w}\right)}
\end{array}\right]^{\mathrm{T}} \mathscr{M}\left[\begin{array}{c}
M \\
I_{\left(n+n_{w}\right)}
\end{array}\right]<0
$$

where

$$
\mathscr{M}=\mathscr{P}_{\mathscr{M}}^{\mathrm{T}} \operatorname{diag}\left(\left[\begin{array}{cc}
X S & Y S+G \\
Y^{\mathrm{T}} S+G^{\mathrm{T}} & Z S
\end{array}\right],-\left[\begin{array}{cc}
Z_{\text {perf }} & Y_{\text {perf }}^{\mathrm{T}} \\
Y_{\text {perf }} & X_{\text {perf }}
\end{array}\right]\right) \mathscr{P}_{\mathscr{M}}
$$

Proof. See Appendix, Section B.

We note that the above conditions are (sufficient) conditions expressed in the form of LMIs on the scaling matrices $S$ and $G$.

\subsection{Synthesis result}

The main result is summarized in the following theorem. To the matrices $X, Y, Z$ and $X_{\text {perf }}$, $Y_{\text {perf }}, Z_{\text {perf }}$, we associate matrices $\tilde{X}, \tilde{Y}, \tilde{Z}$ and $\tilde{X}_{\text {perf }}, \tilde{Y}_{\text {perf }}, \tilde{Z}_{\text {perf }}$ such that

$$
\left[\begin{array}{ll}
X & Y \\
Y^{\mathrm{T}} & Z
\end{array}\right]\left[\begin{array}{cc}
-\tilde{Z} & \tilde{Y}^{\mathrm{T}} \\
\tilde{Y} & -\tilde{X}
\end{array}\right]=I, \quad\left[\begin{array}{cc}
X_{\text {perf }} & Y_{\text {perf }} \\
Y_{\text {perf }} & Z_{\text {perf }}
\end{array}\right]\left[\begin{array}{cc}
-\tilde{Z}_{\text {perf }} & \tilde{Y}_{\text {perf }}^{\mathrm{T}} \\
\tilde{Y}_{\text {perf }} & -\tilde{X}_{\text {perf }}
\end{array}\right]=I
$$

(The conditions in Definition 2.1 ensure the existence of such matrices.)

The following theorem is proven in the Appendix. This control result is derived from the analysis, Theorem 3.2.

\section{Theorem 3.1}

If there exist $S, T \in \underline{\mathbf{S}}(\boldsymbol{\Delta})$, and $G, H \in \underline{\mathbf{G}}(\boldsymbol{\Delta})$, such that

$$
\begin{gathered}
M_{y}^{\perp^{\mathrm{T}}}\left[\begin{array}{c}
M \\
I_{\left(n+n_{w}\right)}
\end{array}\right]^{\mathrm{T}} \mathscr{M}\left[\begin{array}{c}
M \\
I_{\left(n+n_{w}\right)}
\end{array}\right] M_{y}^{\perp}<0 \\
M_{y}^{\mathrm{T}^{\perp^{\mathrm{T}}}}\left[\begin{array}{c}
M \\
I_{\left(n+n_{z}\right)}
\end{array}\right]^{\mathrm{T}} \mathscr{N}\left[\begin{array}{c}
M \\
I_{\left(n+n_{z}\right)}
\end{array}\right] M_{u}^{\mathrm{T}^{\perp}}<0
\end{gathered}
$$

where the matrices $\mathscr{M}$ and $\mathscr{N}$ are defined as follows:

$$
\begin{gathered}
\mathscr{M}=\mathscr{P}_{M}^{\mathrm{T}} \operatorname{diag}\left(\left[\begin{array}{cc}
X S & Y S+G \\
Y^{\mathrm{T}} S+G^{\mathrm{T}} & Z S
\end{array}\right],-\left[\begin{array}{cc}
Z_{\text {perf }} & Y_{\text {perf }}^{\mathrm{T}} \\
Y_{\text {perf }} & X_{\text {perf }}
\end{array}\right]\right) \mathscr{P}_{\mathscr{M}} \\
\mathscr{N}=\mathscr{P}_{\mathscr{N}}^{\mathrm{T}} \operatorname{diag}\left(\left[\begin{array}{cc}
\tilde{X} S & \tilde{Y} T+H \\
\tilde{Y}^{\mathrm{T}} T+H^{\mathrm{T}} & \tilde{Z} T
\end{array}\right],-\left[\begin{array}{cc}
\tilde{Z}_{\text {perf }} & \tilde{Y}_{\text {perf }}^{\mathrm{T}} \\
\tilde{Y}_{\text {perf }} & \tilde{X}_{\text {perf }}
\end{array}\right]\right) \mathscr{P}_{\mathscr{N}}
\end{gathered}
$$

and if in addition the matrices $S_{i}, T_{i}, G_{i}, H_{i}$ satisfy the class-dependent constraints listed in Table IV, then there exists a robust gain-scheduled controller (in the sense of Definition 2.2) that solves the general control problem. 
Table IV. Table of class-dependent constraints in Theorem 3.1

\begin{tabular}{|c|c|c|}
\hline Class of $\Delta_{i}$ & Structure of $\Delta_{K i}$ & Additional constraint \\
\hline $\mathscr{C}_{1}$ & & {$\left[\begin{array}{cc}S_{i} & I \\
I & T_{i}\end{array}\right] \geqslant 0 \quad$ and } \\
\hline$\left(\Delta_{i}=g_{i} I_{n_{i}}\right)$ & $\Delta_{K i}=g_{i} I_{k_{i}}$ & $\operatorname{rank}\left[\begin{array}{cc}S_{i} & I \\
I & T_{i}\end{array}\right]=k_{i}+n_{i}, k_{i} \leqslant n_{i}$ \\
\hline $\mathscr{C}_{2} \cup \mathscr{C}_{4}$ & $\Delta_{K i}=I_{0}$ or $\quad \Delta_{K i}=\Delta_{i}$ & $s_{i} t_{i}=1$ or $\left[\begin{array}{cc}s_{i} & 1 \\
1 & t_{i}\end{array}\right]>0$ \\
\hline $\mathscr{C}_{3}$ & & $\operatorname{rank}\left[\begin{array}{cc}S_{i}+\Gamma_{i}^{-1} G_{i} & I \\
I & T_{i}+\Gamma_{i} H_{i}\end{array}\right]=k_{i}+n_{i}$ \\
\hline$\left(\Delta_{i}=\delta_{i} I_{n_{i}}\right)$ & $\Delta_{K i}=\delta_{i} I_{k_{i}}$ & with $k_{i} \leqslant n_{i}$ and $\Gamma_{i}=\left(Y_{i}^{Y} Y_{i}-X_{i} Z_{i}\right)^{1 / 2}$ \\
\hline $\mathscr{C}_{5} \cup \mathscr{C}_{6}$ & $\Delta_{K_{i}}=I_{0}$ & $S_{i} T_{i}=I$ \\
\hline
\end{tabular}

Remark. If one considers the case when all the matrices $X, Y, Z, X$ perf $, Y_{\text {perf }}, Z_{\text {perf }}$ are diagonal, the above conditions can be dramatically simplified (see Reference 29).

Checking the conditions. Testing the above sufficient conditions for robust control reduces to finding the matrices $S, T \in \underline{\mathbf{S}}(\boldsymbol{\Delta})$ and $G, H \in \underline{\mathbf{G}}(\boldsymbol{\Delta})$ that satisfy LMIs, with possibly additional rank conditions.

Note that $S, T, G, H$ appear linearly in the conditions (12), (13) and in the condition

$$
\left[\begin{array}{cc}
S_{i} & I \\
I & T_{i}
\end{array}\right] \geqslant 0
$$

The above kind of conditions are LMI conditions.

Non-convexities arise in some conditions in the theorem, depending on the perturbation class considered and required controller structure. For example, $S, T, G, H$ appear nonlinearly in the equality condition $S_{i} T_{i}=I$ corresponding to the classes $\mathscr{C}_{5}, \mathscr{C}_{6}$. Similarly, the condition:

$$
\operatorname{rank}\left[\begin{array}{cc}
S_{i}+\Gamma_{i}^{-1} G_{i} & I \\
I & T_{i}+\Gamma_{i} H_{i}
\end{array}\right]=k_{i}+n_{i}
$$

with $\Gamma_{i}=\left(Y_{i}^{\mathrm{T}} Y_{i}-X_{i} Z_{i}\right)^{1 / 2}$ or $\Gamma_{i}=0$ is not convex in the variables, if the required controller structure imposes $k_{i}<n_{i}$.

The consequences are the following. If one considers an interconnection of linear subsystems and if a controller is sought, with $\Delta_{K}=\Delta$, one just needs to check the conditions (12), (13) and (14). The problem is then just a set of LMI constraints, which are easily solved, see e.g., References 34-36. If one considers an interconnection of linear systems and if a controller is sought, with $\Delta_{K}$ constrained to have a 'partial' copy of $\Delta$, then non-convex conditions arise. Non-convexity also appears when one considers the control of an interconnected system with nonlinear operators (classes $\mathscr{C}_{5}, \mathscr{C}_{6}$ ). 
The non-convex conditions in the previous theorem always take the form of a rank constraint imposed on a matrix affine in the decision variables. Several algorithms have been proposed for this kind of problem. In the case $G=0$, El Ghaoui, Oustry and Ait Rami have proposed an efficient heuristic approach to this class of optimization problem. ${ }^{37}$ (Other heuristics were proposed, see e.g., Reference 38.) A generalization of the heuristic of Reference 37 to the case when $G \neq 0$ will be proposed in a forthcoming paper.

Example. A solution to the nominal gain-scheduling problem is given by a direct application of Theorem 3.1.

\section{Corollary 3.1}

There exists a controller of the form (9) which stabilizes the system (1) and ensures for the closed-loop system an $\mathscr{L}_{2}$-gain less then $\gamma$, if there exist matrices $P, Q \in \mathscr{S}(n), S_{i}, T_{i} \in \mathscr{S}\left(n_{i}\right)$ and $G_{i}, H_{i} \in \mathscr{G}\left(n_{i}\right)$ such that

$$
\begin{aligned}
& S=\operatorname{diag}\left(P, \operatorname{diag}\left(S_{1}, \ldots, S_{r}\right)\right), \quad T=\operatorname{diag}\left(Q, \operatorname{diag}\left(T_{1}, \ldots, T_{r}\right)\right) \\
& G=\operatorname{diag}\left(0_{n}, \operatorname{diag}\left(G_{1}, \ldots, G_{r}\right)\right), H=\operatorname{diag}\left(0_{n}, \operatorname{diag}\left(H_{1}, \ldots, H_{r}\right)\right)
\end{aligned}
$$

and

$$
\text { (12), (13), } \quad\left[\begin{array}{ll}
P & I \\
I & Q
\end{array}\right] \geqslant 0
$$

Note that all the conditions in the corollary are LMIs. The link between this result and related ones is discussed in Section 4.1.

It is instructive to see how the above conditions are modified if some parameters are not measured. Assume, for instance, that the first parameter $\delta_{1}(t)$ is no longer measured. Then the sought controller has the form:

$$
u=\mathscr{F}_{u}\left(K, \operatorname{diag}\left(\int I_{n}, \delta_{2} I_{n_{2}}, \ldots, \delta_{r}(t) I_{n_{r}}\right)\right) y
$$

From Table 4, such a law of control exists if the conditions of the Corollary 3.1 are satisfied, and if, in addition, the first blocks $S_{1}, T_{1}, G_{1}, H_{1}$ of $S, T, G, H$ satisfy

$$
\operatorname{rank}\left[\begin{array}{cc}
S_{1}+\frac{1}{b_{1}-a_{1}} G_{1} & I \\
I & T_{1}+\left(b_{1}-a_{1}\right) H_{1}
\end{array}\right]=n_{1}
$$

The problem is no longer convex, due to the above rank condition. More on this kind of problem is given in Section 4.2.

\section{APPLICATIONS}

In the applications we consider (except the last one), a controller is designed to ensure, for the closed-loop system, an $\mathscr{L}_{2}$ gain attenuation less than $\gamma$. Thus, in the sequel, we set $X_{\text {perf }}=\gamma^{2} I$, $Y_{\text {perf }}=0, Z_{\text {perf }}=-I, \tilde{X}_{\text {perf }}=I, \tilde{Y}_{\text {perf }}=0, \tilde{Z}_{\text {perf }}=-\gamma^{-2} I$. 


\subsection{Nominal gain-scheduling problem}

Corollary 3.1 shows how to solve the gain-scheduling problem using LMI (sufficient) conditions. We now discuss the link between our result and previous ones, precisely those found by Packard, ${ }^{4}$ Apkarian and Gahinet, ${ }^{5}$ and Helmersson. ${ }^{11}$

Applying Corollary 3.1 with the parameter intervals $[-1,1]$, and setting the skew-symmetric scaling matrices $G_{i}$ to zero, we recover the results in Reference 5. It turns out that adding a skew-symmetric variable reduces the conservatism of these conditions, as evidenced by numerical experiments given in Section 5. The results of Reference 5 can be interpreted as a continuoustime counterpart of the results of Reference 4. Our approach can be adapted to the discrete-time case considered by Reference 4. Applying Theorem 3.1 with $X=I, Y=0$ and $Z=-I$, the result of Reference 11 are obtained. Moreover, setting the skew-symmetric scaling matrices $G_{i}$ to zero, the results of Reference 4 are recovered.

\subsection{Robust gain-scheduling problem}

The previous result can be extended to consider a more realistic model for the gain-scheduling problem. In this section, we focus on the two following points.

- Usually, dynamical uncertainties appear in different points of a system: some parts of the dynamics of the system itself, or of the actuators and the sensors are never completely modelled. Since gain-scheduled control is usually used to control nonlinear systems, a natural model for these uncertainties is the class $\mathscr{C}_{6}$ of MIMO operators $\Delta_{i}$. We can assume that they have an $\mathscr{L}_{2}$-gain less than one.

- The time-varying parameters $\delta(t)$ are measured with a noise $\delta_{e}(t)$. We assume that this noise is deterministic and that its magnitude is bounded in magnitude by a positive scalar $v$. The measured parameter is thus $\delta_{m}(t)=\delta(t)+\delta_{e}(t)$. In the model of the plant, $\delta(t)$ is in the sequel replaced by $\delta_{m}(t)-\delta_{e}(t), \delta_{e}$ can be considered as an unknown physical parameter, with $\left|\delta_{e}\right| \leqslant v$.

To avoid cumbersome notation, we assume that there is one dynamical uncertainty $\Delta_{n}$ and one measured parameter $\delta(t)$ belonging to $[a, b]$ with $a \leqslant 0$ and $b \geqslant 0$ with a noise $\delta_{e}(t)$. The plant can be written in the following format:

with

$$
\left[\begin{array}{c}
q \\
z \\
\hline y
\end{array}\right]=\left[\begin{array}{c|c}
M & M_{u} \\
\hline M_{y} & 0
\end{array}\right]\left[\begin{array}{c}
p \\
w \\
\hline u
\end{array}\right], \quad p=\Delta q
$$

$$
\Delta(\delta(t))=\operatorname{diag}\left(\int I_{n}, \Delta_{n}, \delta_{m} I_{r}, \delta_{e} I_{r}\right)
$$

Thus, the matrices $X, Y$ and $Z$ are chosen as:

$$
\begin{aligned}
& X=\operatorname{diag}\left(0_{n}, I,-2 a b I_{r}, I_{r}\right) \\
& Y=\operatorname{diag}\left(I_{n}, 0,(a+b) I_{r}, 0\right) \\
& Z=\operatorname{diag}\left(0_{n},-I,-2 I_{r},-v^{2} I_{r}\right)
\end{aligned}
$$


A solution of the robust gain-scheduling problem is given by a direct application of Theorem 3.1:

\section{Corollary 4.1}

There exists a controller $u=\mathscr{F}_{u}\left(K, \operatorname{diag}\left(\int I_{n}, \delta_{m} I_{r}\right)\right) y$ where $K$ is a real matrix, which stabilizes the system (16) and ensures for the closed-loop system an $\mathscr{L}_{2}$-gain less than $\gamma$, if there exist symmetric matrices $P, Q \in \mathscr{S}(n), S_{m}, S_{e}, T_{m}, T_{e} \in \mathscr{S}(r)$, skew-symmetric matrices $G_{m}, G_{e}, H_{m}$, $H_{e} \in \mathscr{G}(r)$ and two positive scalars $s_{n}$ and $t_{n}$ such that

$$
\begin{aligned}
& S=\operatorname{diag}\left(P, s_{n} I, S_{m}, S_{e}\right), \quad T=\operatorname{diag}\left(Q, t_{n} I, T_{m}, T_{e}\right) \\
& G=\operatorname{diag}\left(0_{n}, 0, G_{m}, G_{e}\right), \quad H=\operatorname{diag}\left(0_{n}, 0, H_{m}, H_{e}\right)
\end{aligned}
$$

and

$$
\begin{gathered}
(12), \quad(13), \quad\left[\begin{array}{cc}
P & I \\
I & Q
\end{array}\right] \geqslant 0 \\
\text { and } S_{n} t_{n}=1, \quad\left(S_{e}+v^{-1} G_{e}\right)\left(T_{e}+v H_{e}\right)=I_{r}
\end{gathered}
$$

Note that the last two conditions are not convex.

\subsection{Anti-windup control}

We consider a linear time-invariant, continuous-time system $G$ with input 'saturation functions':

$$
\begin{aligned}
& \dot{x}=A x+B_{w} w+\sum_{i=1}^{n_{u}} B_{u_{i}}\left(u_{i}-g_{i}\left(u_{i}\right)\right) \\
& z=C_{z} x+D_{z w} w+\sum_{i=1}^{n_{u}} D_{z u_{i}}\left(u_{i}-g_{i}\left(u_{i}\right)\right) \\
& y=C_{y} x+D_{y w} w
\end{aligned}
$$

The functions $g_{i}$ are static, time-invariant nonlinearities which belong to $\mathscr{C}_{6}$ and are $\{0,1,-2\}$ dissipative. Furthermore, $g_{i}\left(u_{i}\right) / u_{i}$ is assumed to be bounded and $g(0) / 0=0$. The $g_{i}$ 's are generally dead zone nonlinearities (see Figure 2).

We seek a controller ensuring (i) stability (ii) an $\mathscr{L}_{2}$-gain attenuation between $w$ and $z$ despite the saturations. The first step is to model the plant as the interconnection of operators, with

$$
\left[\begin{array}{c|c}
M & M_{u} \\
\hline M_{y} & 0
\end{array}\right]=\left[\begin{array}{ccc|c}
A & -B_{u} & B_{w} & B_{u} \\
0 & 0 & 0 & I_{n_{u}} \\
C_{z} & -D_{z u} & D_{z w} & D_{z u} \\
\hline C_{y} & 0 & D_{y w} & 0
\end{array}\right]
$$

Two different models of $\Delta$ are possible:

- considering that $g_{i}$ is a nonlinearity: $\Delta=\operatorname{diag}\left(\int I_{n}, \operatorname{diag}\left(g_{1}(\cdot), \ldots, g_{n_{u}}(\cdot)\right)\right)$.

- considering that $g_{i}\left(u_{i}\right)=\left(g_{i}\left(u_{i}\right) / u_{i}\right) u_{i}$ (multiplication by the time-varying gain $\left.g_{i}\left(u_{i}(t)\right) / u_{i}(t)\right)$ : $\Delta=\operatorname{diag}\left(\int I_{n}, \operatorname{diag}\left(g_{1}\left(u_{1}\right) / u_{1}, \ldots, g_{n_{u}}\left(u_{n_{u}}\right) / u_{n_{u}}\right)\right)$. 


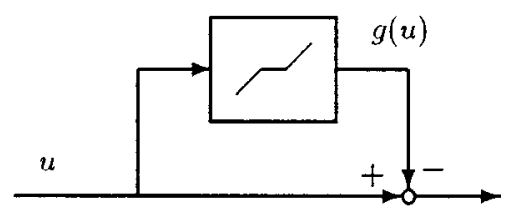

Figure 2. Input nonlinearity

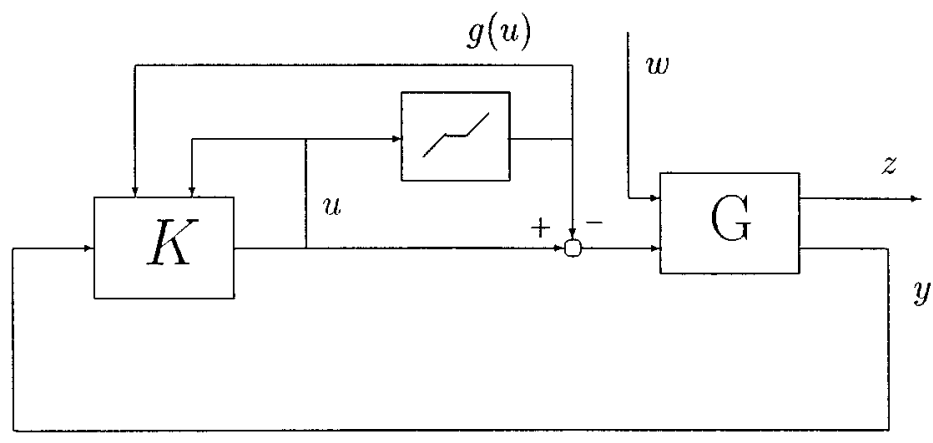

Figure 3. Anti-windup control

These two models are equivalent. ${ }^{39}$ But, if we consider the first one, as $g_{i}$ is nonlinear, applying the Theorem 3.1 leads to a non-convex constraint. Note that $g_{i}$ is known and the command input $u_{i}$ is perfectly 'measured' in real time. Then, with $\delta_{i}(t)=g_{i}\left(u_{i}(t)\right) / u_{i}(t)$, this problem is similar to the gain-scheduling problem is thus obtained with the second model. The obtained controller has the form $u=\mathscr{F}_{u}\left(K, \operatorname{diag}\left(\int I_{n}, \operatorname{diag}\left(g_{1}\left(u_{1}\right) / u_{1}, \ldots, g_{n_{u}}\left(u_{n_{u}}\right) / u_{n_{u}}\right)\right) y\right.$ (see Figure 3$)$.

Theorem 3.1 is applied with $X=\operatorname{diag}\left(0_{n}, 0_{n_{u}}\right), Y=\operatorname{diag}\left(I_{n}, I_{n_{u}}\right), Z=\operatorname{diag}\left(0_{n},-2 I_{n_{u}}\right)$.

The solution of the anti-windup control problem is given by a direct application of Theorem 3.1.

\section{Corollary 4.2}

There exists a controller

$$
u=\mathscr{F}_{u}\left(K, \operatorname{diag}\left(\int I_{n}, \operatorname{diag}\left(\frac{g_{1}\left(u_{1}(t)\right)}{u_{1}(\mathrm{t})}, \ldots, \frac{g_{n_{u}}\left(u_{n_{u}}(t)\right)}{u_{n_{u}}(t)}\right)\right)\right) y
$$

where $K$ is an $\left(n+n_{u}+n_{u}\right) \times\left(n+n_{u}+n_{y}\right)$ real matrix, which stabilizes the system (18) and ensures to the closed-loop system an $\mathscr{L}_{2}$-gain less than $\gamma$ if there exist, $P, Q \in \mathscr{S}(n), n_{u}$ strictly positive scalars $s_{1}, \ldots, s_{n_{u}}$ and $n_{u}$ strictly positive scalars $t_{1}, \ldots, t_{n_{u}}$ such that

$$
S=\operatorname{diag}\left(P, \operatorname{diag}\left(s_{1}, \ldots, S_{n_{u}}\right)\right), T=\operatorname{diag}\left(Q, \operatorname{diag}\left(t_{1}, \ldots, t_{n_{u}}\right)\right)
$$

and

(12), (13), $\quad\left[\begin{array}{ll}S & I \\ I & T\end{array}\right] \geqslant 0$ 


\subsection{Control of nonlinear rational systems}

The control of rational systems was considered in the paper. ${ }^{27}$ Here, we introduce less conservative conditions for the output-feedback control of this class of nonlinear systems.

We consider a nonlinear, time-invariant, continuous-time system with an equilibrium point at $x=0$ :

$$
\begin{aligned}
& \dot{x}=\mathbf{A}(x) x+\mathbf{B}_{w}(x) w+\mathbf{B}_{u}(x) u \\
& z=\mathbf{C}_{z}(x) x+\mathbf{D}_{z w}(x) w+\mathbf{D}_{z u}(x) u \\
& y=\mathbf{C}_{y}(x) x+\mathbf{D}_{y w}(x) w
\end{aligned}
$$

Here, $\mathbf{A}, \mathbf{B}_{u}, \ldots$ are (multivariable, well-posed) rational functions of $x_{i}$, with $i \in \mathscr{I} . \mathscr{I}$ is a subset of $\{1, \ldots, n\}$, which corresponds to the state variables appearing nonlinearly in the state-space equations of the system.

The above system can be rewritten in the LFT format (2) with appropriate matrices $M, M_{u}$, $M_{y}$ and $\Delta$ given by (5), where $\delta_{i}$ replaced by $x_{i}$ with $i \in \mathscr{I}$. (The variables that appear linearly in the state-space equations (20) do not appear in the matrix $\Delta$.)

We seek a controller which ensures for the closed-loop system (i) stability and (ii) an $\mathscr{L}_{2}$-gain attenuation of $\gamma$, for trajectories with zero initial condition and $\|w\| \leqslant 1$. For this particular case, we are able to introduce a condition which guarantees that every $x_{i}$ with $i \in \mathscr{I}$ is bounded in modulus by a positive scalar $\sigma$ (for details see the paper $^{27}$ ). (Here, $\sigma$ can be considered as a design parameter.)

Denote by $\mathscr{I}_{2}$ the subset of integers in $\mathscr{I}$ corresponding to measured state variables. (This set defines the state variables that appear nonlinearly in the state-space equations and are measured.) Applying Theorem 3.1 with $X=\operatorname{diag}\left(0_{n}, I\right), Y=\operatorname{diag}\left(I_{n}, 0\right), Z=\operatorname{diag}\left(0_{n},-\sigma^{2} I\right)$ results in the following corollary.

\section{Corollary 4.3}

There exists a controller

$$
u=\mathscr{F}_{u}\left(K, \operatorname{diag}\left(\int I_{n}, \operatorname{diag}\left(x_{i}(t) I_{n_{i}}\right)_{i \in \mathscr{I}_{2}}\right)\right) y
$$

with $K$ a constant real matrix, which stabilizes the system (20) and ensures to the closed-loop system an $\mathscr{L}_{2}$-gain less than $\gamma$ with the initial condition 0 and $\|w\| \leqslant 1$, if there exist a scalar $\sigma>0, P, Q \in \mathscr{S}(n), S_{i}, T_{i} \in \mathscr{S}\left(n_{i}\right)$ and $G_{i}, H_{i} \in \mathscr{G}\left(n_{i}\right)$ with $i \in \mathscr{I}$ such that

$$
\begin{aligned}
& S=\operatorname{diag}\left(P, \operatorname{diag}\left(S_{1}, \ldots, S_{n}\right)\right), \quad T=\operatorname{diag}\left(Q, \operatorname{diag}\left(T_{1}, \ldots, T_{n}\right)\right) \\
& G=\operatorname{diag}\left(0_{n}, \operatorname{diag}\left(G_{1}, \ldots, G_{n}\right)\right), H=\operatorname{diag}\left(0_{n}, \operatorname{diag}\left(H_{1}, \ldots, H_{n}\right)\right)
\end{aligned}
$$

and

(12), (13), $\quad e_{i}^{\mathrm{T}} Q e_{i}<\sigma^{-2} \gamma^{-2}, \quad$ for all $i \in \mathscr{I}$

$$
\left(S_{i}+\Gamma_{i}^{-1} G_{i}\right)\left(T_{i}+\Gamma_{i} H_{i}\right)=I, \quad \text { for } \quad \text { all } i \in \mathscr{I} \backslash \mathscr{I}_{2},\left[\begin{array}{ll}
P & I \\
I & Q
\end{array}\right] \geqslant 0
$$


When $\mathscr{I}=\mathscr{I}_{2}$, that is, when every variable appearing nonlinearly in the state-space equations is measured, then the above conditions are convex (for a given $\sigma$ ).

\subsection{Model reduction problem}

We consider the following stable interconnected system:

$$
z(t)=\mathscr{F}_{u}\left(\left[\begin{array}{ll}
A & B \\
C & D
\end{array}\right], \Delta\right) w(t)
$$

where $\Delta=\operatorname{diag}\left(\Delta_{i}\right)$, with $\Delta_{i}$ belongs to the class $\mathscr{C}_{1}, \mathscr{C}_{2}, \mathscr{C}_{3}$ or $\mathscr{C}_{4}$, and satisfies a given $\{X, Y, Z\}$-dissipative property. We seek to approximate the above system, up to an $\mathscr{L}_{2}$-gain $\gamma$, by a stable interconnected system

$$
z_{r}(t)=\mathscr{F}_{u}\left(\left[\begin{array}{ll}
A_{r} & B_{r} \\
C_{r} & D_{r}
\end{array}\right], \Delta_{r}\right) w(t)
$$

where $\Delta_{r}$ is a partial copy of $\Delta$ and such that

$$
\left\|z-z_{r}\right\| \leqslant \gamma^{2}\|w\|
$$

This problem reduces to find, for the system

$$
\left[\begin{array}{c}
z(t) \\
y(t)
\end{array}\right]=\mathscr{F}_{u}\left(\left[\begin{array}{cc|c}
A & B & 0 \\
C & D & -I \\
\hline 0 & I & 0
\end{array}\right], \Delta\right)\left[\begin{array}{c}
w(t) \\
u(t)
\end{array}\right]
$$

a 'controller'

$$
u(t)=\mathscr{F}_{u}\left(\left[\begin{array}{ll}
A_{r} & B_{r} \\
C_{r} & D_{r}
\end{array}\right], \Delta_{r}\right) y(t)
$$

such that the closed-loop system has an $\mathscr{L}_{2}$-gain between $w$ and $z$ less then $\gamma$. Theorem 3.1 can be then directly applied to obtain the following corollary. As before, we introduce $\tilde{X}, \tilde{Y}, \tilde{Z}$, as defined by (11).

\section{Corollary 4.4}

Assume that there exist $S, T \in \underline{\mathbf{S}}(\boldsymbol{\Delta})$ and $G, H \in \underline{\mathbf{G}}(\boldsymbol{\Delta})$, such that

$$
\begin{gathered}
{\left[\begin{array}{c}
A \\
I_{\left(n+n_{w}\right)}
\end{array}\right]^{\mathrm{T}}\left[\begin{array}{cc}
X S & Y S+G \\
Y^{\mathrm{T}} S+G^{\mathrm{T}} & Z S
\end{array}\right]\left[\begin{array}{c}
A \\
I_{\left(n+n_{w}\right)}
\end{array}\right]<-C^{\mathrm{T} C}} \\
{\left[\begin{array}{c}
A^{\mathrm{T}} \\
I_{\left(n+n_{z}\right)}
\end{array}\right]^{\mathrm{T}}\left[\begin{array}{cc}
\tilde{X} T & \tilde{Y} T+H \\
\tilde{Y}^{\mathrm{T}} T+H^{\mathrm{T}} & \tilde{Z} T
\end{array}\right]\left[\begin{array}{c}
A^{\mathrm{T}} \\
I_{\left(n+n_{z}\right)}
\end{array}\right]<-B B^{\mathrm{T}}}
\end{gathered}
$$

and for $i$ such that $\Delta_{i}$ does not belong to the class $\mathscr{C}_{3}$,

$$
\left[\begin{array}{ll}
S_{i} & \gamma I \\
\gamma I & T_{i}
\end{array}\right] \geqslant 0
$$


Then, there exists a reduced model of the form (23), with $\Delta_{r}=\operatorname{diag}\left(\Delta_{r, 1}, \ldots, \Delta_{r, n}\right)$, where

- if $\Delta_{i}$ is a MIMO operator (classes $\mathscr{C}_{2}$ or $\mathscr{C}_{4}$ ), then: $\Delta_{r, i}=\Delta_{i}$ if $S_{i} T_{i} \neq \gamma^{2} I, \Delta_{r, i}=I_{0}$ otherwise;

- if $\Delta_{i}=\delta_{i} I_{n_{i}}$ with $\delta_{i}$ a SISO operator (classes $\mathscr{C}_{1}$ or $\mathscr{C}_{3}$ ), then $\Delta_{r, i}=\delta_{i} I_{k_{i}}$, where

$$
\operatorname{rank}\left[\begin{array}{cc}
S_{i}+\Gamma_{i}^{-1} G_{i} & \gamma I \\
\gamma I & T_{i}+\Gamma_{i} H_{i}
\end{array}\right]=k_{i}+n_{i}
$$

As in References 11 and 25, the obtained formulation is a non-convex optimization problem. A related formulation was first considered by Beck and co-authors. ${ }^{25,26}$ Here again, our contribution is the introduction of the skew-symmetric scaling $G$. A related improvement was proposed by Herlmersson, ${ }^{11}$ using the passivity framework. Wood and co-authors ${ }^{40}$ considered the model reduction of the system (1). A potentially less conservative formulation is obtained in this specific case: unfortunately the corresponding non-convex optimization problem is infinitedimensional.

\subsection{Control of static systems}

In this section, we define a static system as an interconnected system of the form (2), where the matrix $\Delta$ is considered pointwise dissipative.

\section{Definition 4.1}

A matrix $H$ is said to be $\{X, Y, Z\}$-pointwise dissipative if there exist constant matrices $X>0$ (or $X=0$ ), $Y$ and $Z \leqslant 0$ such that $\Phi=\left[\begin{array}{cc}X & Y \\ Y^{\mathrm{T}} & Z\end{array}\right]$ is a full-rank matrix and $p=H q$ implies

$$
\left[\begin{array}{l}
q \\
p
\end{array}\right]^{\mathrm{T}} \Phi\left[\begin{array}{l}
q \\
p
\end{array}\right] \geqslant 0
$$

The important fact is that to an $\{X, Y, Z\}$-pointwise dissipative matrix can be associated an $\{X, Y, Z\}$-dissipative operator. The consequence is that the discussion of Section 2 and Theorem 3.1 can be straightforwardly adapted to this class of systems.

This framework is classical in the context of the robustness analysis of uncertain, linear time-invariant systems. ${ }^{41,42}$ For instance, it is the framework considered by Helmersson. ${ }^{11}$ As is pointed out in the paper, ${ }^{43}$ the structured robust least squares problem can be formulated in this framework. Finding a solution to this problem can be interpreted as finding a controller for a static system. Another interesting application of this framework is the steady-sate control of uncertain systems. ${ }^{44}$ The conditions obtained in this paper can be straightforwardly adapted to recover known results for this class of systems.

\section{NUMERICAL EXPERIMENTS}

To demonstrate the improvement given by the use of skew-symmetric matrices, we have considered the nominal gain-scheduling problem, and associated synthesis result (Corollary 3.1). We have generated random third-order systems, perturbed by a single (scalar) parameter $\delta$ repeated six times on the diagonal perturbation matrix $\Delta$, and bounded by one in magnitude. (With the notation of (3), $\Delta_{\mathrm{par}}=\delta I_{6}$ ). These systems have two actuators, two measured outputs, one input $w$ and one output $z$. 
For each system, we seek to compute the smallest $\mathscr{L}_{2}$-gain (between $w$ and $z$ ) achievable by gain-scheduled output-feedback for the closed-loop system. We computed two quantities. The first one, denoted $\gamma_{S}$, corresponds to Apkarian's conditions (which are obtained by setting the skew-symmetric variables $G, H$ to zero in our formulation).

To define precisely these two quantities, define

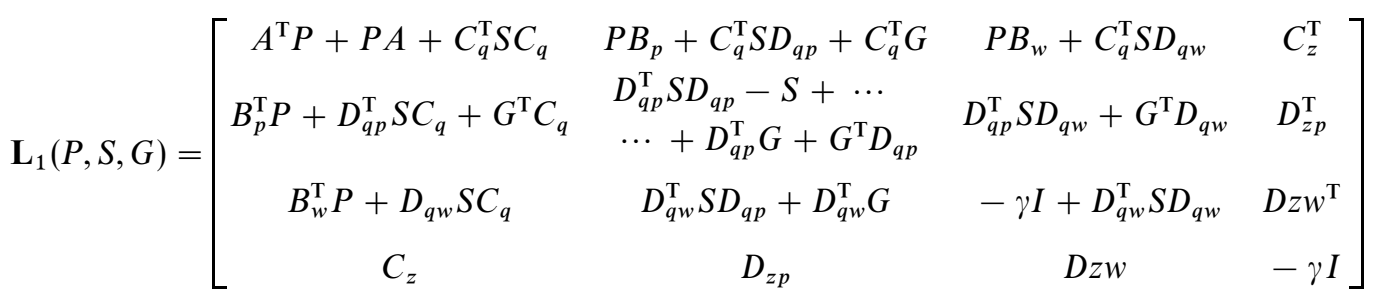

and

$$
\mathbf{L}_{2}(Q, T, H)=\left[\begin{array}{cccc}
A Q+Q A^{\mathrm{T}}+B_{p} T B_{p}^{\mathrm{T}} & Q C_{q}^{\mathrm{T}}+B_{p} T D_{q p}^{\mathrm{T}}+B_{p} H & Q C_{z}^{\mathrm{T}}+B_{p} T D_{z p}^{\mathrm{T}} & B_{w} \\
C_{q} Q+D_{q p} T B_{p}^{\mathrm{T}}+H^{\mathrm{T}} B_{p}^{\mathrm{T}} & D_{q p} T D_{q p}^{\mathrm{T}}-T+\cdots & D_{q p} T D_{z p}^{\mathrm{T}}+H^{\mathrm{T}} D_{z p}^{\mathrm{T}} & D_{q w} \\
C_{z} Q+D_{z p} T B_{p}^{\mathrm{T}} & D_{z p} T D_{q p}^{\mathrm{T}}+D_{z p} H & -\gamma I+D_{z p} T D_{z p}^{\mathrm{T}} & D_{z w} \\
B_{w}^{\mathrm{T}} & D_{q w}^{\mathrm{T}} & D_{z w}^{\mathrm{T}} & -\gamma I
\end{array}\right]
$$

Also, define

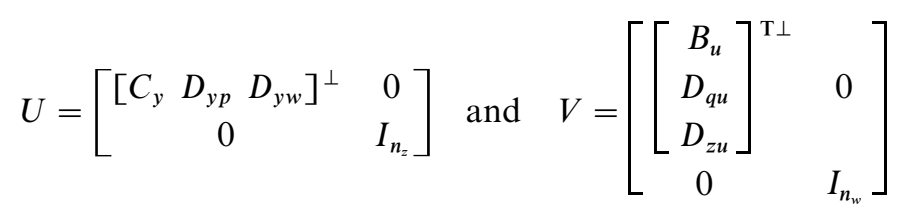

We define $\gamma_{s}$ by

$$
\begin{aligned}
& \gamma_{S}=\text { minimize } \quad \gamma \\
& \text { on } \quad P, Q, S, T \\
& \text { subject to } U^{\mathrm{T}} \mathbf{L}_{1}(P, S, 0) U<0, V^{\mathrm{T}} \mathbf{L}_{2}(Q, T, 0) V<0 \\
& {\left[\begin{array}{ll}
P & I \\
I & Q
\end{array}\right] \geqslant 0, \quad\left[\begin{array}{ll}
S & I \\
I & T
\end{array}\right] \geqslant 0}
\end{aligned}
$$

The second quantity, denoted $\gamma_{S G}$, is obtained by use of skew-symmetric scalings. Precisely,

$$
\begin{aligned}
& \gamma_{S G}=\operatorname{minimize} \quad \gamma \\
& \text { on } \quad P, Q, S, T, G, H \\
& \text { subject to } U^{\mathrm{T}} \mathbf{L}_{1}(P, S, G) U<0, V^{\mathrm{T}} \mathbf{L}_{2}(Q, T, H) V<0 \\
& {\left[\begin{array}{ll}
P & I \\
I & Q
\end{array}\right] \geqslant 0, \quad S>0, T>0}
\end{aligned}
$$




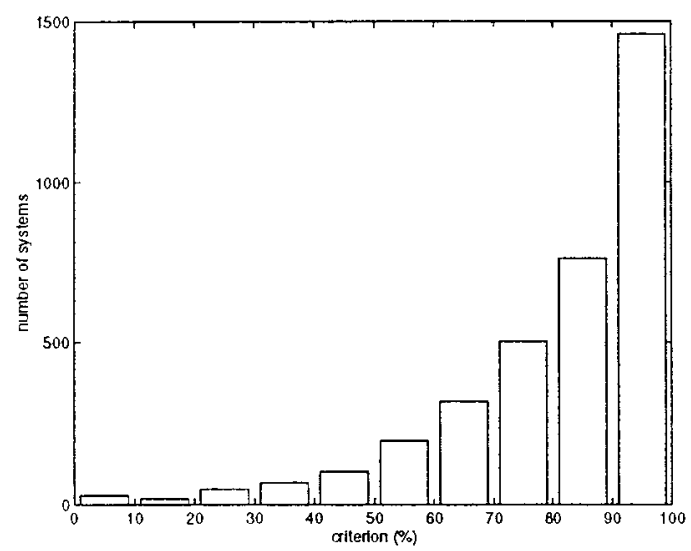

Figure 4. Histogram

These two problems are LMI problems, and can be solved efficiently using available Matlab tool boxes. Our results were obtained via the Matlab interface LMITOOL to the interior-point code SP. ${ }^{34}$

For the sake of comparison, we chose the following criterion to measure the improvement resulting from skew-symmetric scalings:

$$
100 \frac{\gamma_{S}-\gamma_{S G}}{\gamma_{S}}
$$

The results for 3500 randomly generated systems are summarized in the histogram (Figure 4). The $x$-axis represents the different values of the criterion, classified in ten intervals: from $0 \%$ to $10 \%, \ldots, 90 \%$ to $100 \%$. The $y$-axis represents the number of systems for which the criterion is in each interval. The improvement is between $0 \%$ and $100 \%$ and the mean is $80.8 \%$.

\section{CONCLUSION}

This paper presents a general framework for the control of interconnections of time varying and/or nonlinear operators. We derived sufficient conditions for the stability and performance control of such systems. These conditions are also necessary for some classes of operators $\Delta$ (e.g., when the operators $\Delta_{i}$ are dynamic, and only known to be linear time-variant, or nonlinear time-invariant and to satisfy a dissipative property ${ }^{46,47}$ ).

Our main motivation is to provide an attractive solution for the gain-scheduling problem. However, the approach can be applied to several classes of related problems. For the gainscheduling problem, dramatic improvements over the approach proposed by Apkarian and Gahinet ${ }^{5}$ are numerically demonstrated. Some extensions to the multi-objective control problem can be considered, as in the paper. ${ }^{48}$ 


\section{APPENDIX}

The result 3.1 is proved in three steps.

- A partial parametrization of the matrices $X_{p}, Y_{p}$ and $Z_{p}$ such that $\Delta$ is $\left\{X_{p}, Y_{p}, Z_{p}\right\}$-dissipative is proposed. As a matter of fact, if every $\Delta_{i}$ is $\left\{X_{i}, Y_{i}, Z_{i}\right\}$-dissipative then $\Delta$ satisfies several dissipative properties.

- A sufficient condition for the stability and the performance of an interconnected system is proposed. It can be interpreted using graph separation arguments. ${ }^{17,33}$ Consider the interconnection of $M$ and $\Delta$ with $\Delta$ dissipative. The point is to find a condition over $M$ which implies stability and performance for the interconnection.

- Using the previous condition, existence conditions for a controller which achieves stability and performance for the closed-loop system are derived.

\section{A. Parametrization of the dissipative properties of $\Delta$}

In the first part of this section, we prove Lemma 3.1.

Proof of Lemma 3.1. With $p(t)=\Delta(q)(t)$ and by definition of $S$ : the signals $p$ and $q$ are such that:

$$
\sigma_{0}(p, q)=\int_{0}^{T}\left[\begin{array}{l}
q(t) \\
p(t)
\end{array}\right]^{\mathrm{T}}\left[\begin{array}{cc}
X S & Y S \\
Y^{\mathrm{T}} S & Z S
\end{array}\right]\left[\begin{array}{l}
q(t) \\
p(t)
\end{array}\right] \mathrm{d} t \geqslant 0
$$

Furthermore, $q$ and $p$ also verify the following equality:

$$
p(t)^{\mathrm{T}} G q(t)+q(t)^{\mathrm{T}} G^{\mathrm{T}} p(t)=0
$$

This equality leads to:

$$
\sigma_{1}(p, q)=\int_{0}^{T}\left(p(t)^{\mathrm{T}} G q(t)+q(t)^{\mathrm{T}} G^{\mathrm{T}} p(t)\right) \mathrm{d} t=0
$$

Thus, for all scalar $\tau$, the signals $p$ and $q$ satisfy the following inequality:

$$
\sigma_{0}(p, q)+\tau \sigma_{1}(p, q) \geqslant 0
$$

which is equivalent to (after a change of notation $\tau G \rightarrow G$ )

$$
\int_{0}^{T}\left[\begin{array}{l}
q(t) \\
p(t)
\end{array}\right]^{\mathrm{T}}\left[\begin{array}{cc}
X S & Y S+G \\
Y^{\mathrm{T}} S+G & Z S
\end{array}\right]\left[\begin{array}{c}
q(t) \\
p(t)
\end{array}\right] \mathrm{d} t \geqslant 0
$$

Let us now explicit the structure of the multipliers $S$ and $G$.

Scaling $S$. If $\Delta_{i}$ is a linear full block (classes $\mathscr{C}_{2}, \mathscr{C}_{4}$ ) then for any scalar $\lambda \neq 0, \Delta_{i}=\lambda \Delta_{i} \lambda^{-1}$. Thus, $S_{i}=\lambda^{2} I_{n_{i}}$. if $\Delta_{i}$ is a repeated block (classes $\mathscr{C}_{1}, \mathscr{C}_{3}$ ) then for any invertible matrix $W, \Delta_{i}=W^{-1} \Delta_{i} W$. Thus, $S_{i}=W^{\mathrm{T}} W$

Now, if $\Delta_{i}$ is nonlinear (classes $\left.\mathscr{C}_{5}, \mathscr{C}_{6}\right)$ and $\left\{X_{i}, Y_{i}, Z_{i}\right\}$ dissipative then for any scalar $\lambda \neq 0$ :

$$
\int_{0}^{T}\left[\begin{array}{l}
q(t) \\
p(t)
\end{array}\right]^{\mathrm{T}}\left[\begin{array}{ll}
\lambda^{2} X_{i} & \lambda^{2} Y_{i} \\
\lambda^{2} Y_{i}^{\mathrm{T}} & \lambda^{2} Z_{i}
\end{array}\right]\left[\begin{array}{l}
q(t) \\
p(t)
\end{array}\right] \mathrm{d} t \geqslant 0
$$

Thus, $\lambda \Delta_{i}\left(\lambda^{-1}\right)$ is in the same class as $\Delta_{i}$ and is $\left\{X_{i}, Y_{i}, Z_{i}\right\}$ dissipative.

Scaling G. Consider the case when $\Delta_{i}=\delta I_{n_{i}}$ with $\delta=\delta_{R}+j \delta_{I}$. As $G$ is such that $p(j \omega)^{*} G q(j \omega)$ $+q(j \omega)^{*} G^{\mathrm{T}} p(j \omega)=0$, if $\delta_{R} \neq 0$ and $\delta_{I}=0$ then $G=-G^{\mathrm{T}}$. 


\section{B. Analysis (proof of Theorem 3.2)}

Split the matrix $M$ as

$$
\left[\begin{array}{l}
q(t) \\
z(t)
\end{array}\right]=\left[\begin{array}{l}
M_{\Delta} \\
M_{z}
\end{array}\right]\left[\begin{array}{l}
p(t) \\
w(t)
\end{array}\right] \text { and } M_{\Delta}=\left[\begin{array}{ll}
M_{\Delta 1} & M_{\Delta 2}
\end{array}\right]
$$

where $z$ and $w$ are the output and the input of interest, and $q$ and $p$ are the signals connecting $M$ to the operator $\Delta$.

The proof has three different steps.

- The system is assumed to be stable. We first prove that dissipative performance is ensured if the LMI (10) holds.

- The system is assumed to be well posed (that is, there exist unique $p, q$ and $z$ which depend causally on $w$ ). We then prove that the condition (10) also ensures the stability.

- The third step consists of proving the well posedness from the condition (10).

B.1. Performance. The system is $\left\{X_{\text {perf }}, Y_{\text {perf }}, Z_{\text {perf }}\right\}$ dissipative if for all signals $z, w, q, p$, belonging to $\mathscr{L}_{2}$ and such that:

$$
\left[\begin{array}{l}
q(t) \\
z(t)
\end{array}\right]=\left[\begin{array}{l}
M_{\Delta} \\
M_{z}
\end{array}\right]\left[\begin{array}{l}
p(t) \\
w(t)
\end{array}\right]
$$

and

$$
\int_{0}^{+\infty}\left[\begin{array}{c}
q(t) \\
p(t)
\end{array}\right]^{\mathrm{T}}\left[\begin{array}{cc}
X S & Y S+G \\
Y^{\mathrm{T}} S+G & Z S
\end{array}\right]\left[\begin{array}{c}
q(t) \\
p(t)
\end{array}\right] \mathrm{d} t \geqslant 0
$$

the following integral quadratic constraint holds:

$$
\int_{0}^{+\infty}\left[\begin{array}{l}
z(t) \\
w(t)
\end{array}\right]^{\mathrm{T}}\left[\begin{array}{ll}
Z_{\text {perf }} & Y_{\text {perf }}^{\mathrm{T}} \\
Y_{\text {perf }} & X_{\text {perf }}
\end{array}\right]\left[\begin{array}{c}
z(t) \\
w(t)
\end{array}\right] \mathrm{d} t \geqslant 0
$$

Then, using the decomposition of the matrix $M$ and the equality (30):

$$
(32) \Leftrightarrow \sigma_{0}(p, w)>0
$$

with

$$
\sigma_{0}(p, w)=\int_{0}^{+\infty}\left[\begin{array}{l}
p(t) \\
w(t)
\end{array}\right]^{\mathrm{T}}\left[\begin{array}{c}
M_{z} \\
{\left[\begin{array}{ll}
0 & I
\end{array}\right]}
\end{array}\right]^{\mathrm{T}}\left[\begin{array}{cc}
X_{\text {perf }} & Y_{\text {perf }} \\
Y_{\text {perf }}^{\mathrm{T}} & Z_{\text {perf }}
\end{array}\right]\left[\begin{array}{c}
M_{z} \\
{\left[\begin{array}{ll}
0 & I
\end{array}\right]}
\end{array}\right]\left[\begin{array}{c}
p(t) \\
w(t)
\end{array}\right] \mathrm{d} t
$$

and

$$
(31) \Leftrightarrow \sigma_{1}(p, w)>0
$$

with

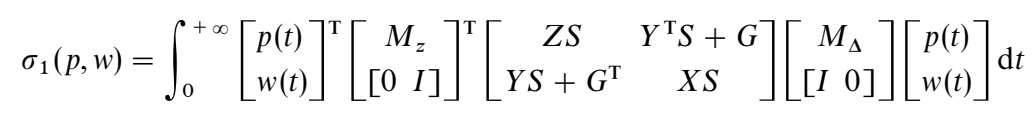

Using the $\mathscr{S}$-procedure, ${ }^{49} \sigma_{0}(p, w)$ is positive for all the signals $p$ and $w$ such that $\sigma_{1}(p, w)>0$ if and only if there exists a positive scalar $\tau$, such that: $-\sigma_{0}(p, w)+\tau \sigma_{1}(p, w) \leqslant 0$ which is equivalent to (after introducing $M$ and changing the notation $\tau S \rightarrow S$ and $\tau G \rightarrow G$ ) and condition (10).

B.2. Stability. We need to prove that the stability of the feedback interconnection of $\Delta$ with $M_{\Delta 1}$ is ensured by the condition (10). More precisely, the condition (10) implies the condition:

$$
\left[\begin{array}{c}
M_{\Delta 1} \\
I_{n}
\end{array}\right]^{\mathrm{T}}\left[\begin{array}{cc}
X S & Y S+G \\
Y^{\mathrm{T}} S+G^{\mathrm{T}} & Z S
\end{array}\right]\left[\begin{array}{c}
M_{\Delta 1} \\
I_{n}
\end{array}\right]<0
$$




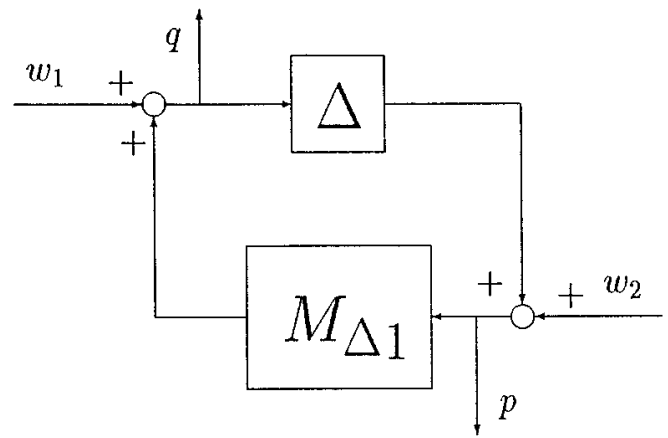

Figure 5. $M_{\Delta 1}-\Delta$ associated system

We now prove that this condition ensures the stability of the loop. Without loss of generality, we assume that $S=I$ and $G=0$. Consider the dissipativity inequality describing $\Delta$ : for all signals $q \in \mathscr{L}_{2 e}$ and $p \in \mathscr{L}_{2 e}$ and $T \in \mathbf{R}^{+}$:

$$
\int_{0}^{\mathrm{T}}\left[\begin{array}{l}
q \\
p
\end{array}\right]^{\mathrm{T}}\left[\begin{array}{cc}
X & Y \\
Y^{\mathrm{T}} & Z
\end{array}\right]\left[\begin{array}{l}
q \\
p
\end{array}\right] \geqslant 0
$$

Using the notation of Figure 5, for all $w_{1}$ and $w_{2}$ in $\mathscr{L}_{2}$, the system is stable if $q$ and $p$ are also in $\mathscr{L}_{2}$. Since the system is assumed to be well posed, $q$ and $p$ belong to $\mathscr{L}_{2 e}$.

First, owing to the characterization of $\Delta$,

$$
\int_{0}^{T}\left(q^{\mathrm{T}} X q+q^{\mathrm{T}} Y\left(p-w_{2}\right)+\left(p-w_{2}\right)^{\mathrm{T}} Y^{\mathrm{T}} q+\left(p-w_{2}\right)^{\mathrm{T}} Z\left(p-w_{2}\right)\right) \mathrm{d} t \geqslant 0
$$

Condition (35) implies that there exists an $\varepsilon>0$ such that

$$
\left[\begin{array}{c}
M_{\Delta 1} \\
I
\end{array}\right]^{\mathrm{T}}\left[\begin{array}{cc}
X & Y \\
Y^{\mathrm{T}} & Z
\end{array}\right]\left[\begin{array}{c}
M_{\Delta 1} \\
I
\end{array}\right] \leqslant-\varepsilon\left(M_{\Delta 1}^{\mathrm{T}} M_{\Delta 1}+I\right)
$$

This leads, for all $T$, to:

$$
\begin{aligned}
& \int_{0}^{T}\left(\left(q-w_{1}\right)^{\mathrm{T}} X\left(q-w_{1}\right)+\left(q-w_{1}\right)^{\mathrm{T}} Y p+p^{\mathrm{T}} Y^{\mathrm{T}}\left(q-w_{1}\right)+p^{\mathrm{T}} Z p\right) \mathrm{d} t \leqslant \cdots \\
& \quad \cdots-\varepsilon \int_{0}^{T}\left(\left(q-w_{1}\right)^{\mathrm{T}}\left(q-w_{1}\right)+p^{\mathrm{T}} p\right) \mathrm{d} t
\end{aligned}
$$

The conditions (36) and (37) imply that:

$$
\begin{gathered}
\int_{0}^{T}\left(\varepsilon q^{\mathrm{T}} q+q^{\mathrm{T}}\left(Y w_{2}-(X+\varepsilon I) w_{1}\right)+\left(Y w_{2}-(X+\varepsilon I) w_{1}\right)^{\mathrm{T}} q+\varepsilon p^{\mathrm{T}} p+p^{\mathrm{T}}\left(Z w_{2}-Y w_{1}\right)\right) \mathrm{d} t \leqslant \cdots \\
\cdots \int_{0}^{T}\left(w_{2}^{\mathrm{T}} Z w_{2}-w_{1}^{\mathrm{T}}(X+\varepsilon I) w_{1}\right) \leqslant 0
\end{gathered}
$$

as $X \geqslant 0$ and $Z \leqslant 0$. Using square completions, this leads, for all $T$, to:

$$
\begin{aligned}
\int_{0}^{T} & \varepsilon\left(q+\frac{1}{\varepsilon}\left(Y w_{2}-(X+\varepsilon I) w_{1}\right)^{\mathrm{T}}\left(q+\frac{1}{\varepsilon}\left(Y w_{2}-(X+\varepsilon I) w_{1}\right)+\cdots\right.\right. \\
& \quad \cdots \varepsilon\left(p+\frac{1}{\varepsilon}\left(Z w_{2}-Y w_{1}\right)\right)^{\mathrm{T}}\left(p+\frac{1}{\varepsilon}\left(Z w_{2}-Y w_{1}\right)\right) \leqslant \cdots \\
& \cdots \frac{1}{\varepsilon}\left(Z w_{2}-Y w_{1}\right)^{\mathrm{T}}\left(Z w_{2}-Y w_{1}\right)+\frac{1}{\varepsilon}\left(Y w_{2}-(X+\varepsilon I) w_{1}\right)^{\mathrm{T}}\left(Y w_{2}-(X+\varepsilon I) w_{1}\right)
\end{aligned}
$$


Using the triangle inequality, for all $T$ :

$$
\begin{aligned}
& \|q\|_{\mathrm{T}} \leqslant \frac{1}{\varepsilon}\left\|Z w_{2}-Y w_{1}\right\|_{\mathrm{T}}+\frac{2}{\varepsilon}\left\|Y w_{2}-(X+\varepsilon I) w_{1}\right\|_{\mathrm{T}} \\
& \|p\|_{\mathrm{T}} \leqslant \frac{1}{\varepsilon}\left\|Z w_{2}-Y w_{1}\right\|_{\mathrm{T}}+\frac{2}{\varepsilon}\left\|Y w_{2}-(X+\varepsilon I) w_{1}\right\|_{\mathrm{T}}
\end{aligned}
$$

Thus, if the inputs $w_{1}$ and $w_{2}$ are in $\mathscr{L}_{2}$ then $q$ and $p$ are in $\mathscr{L}_{2}$, too. The closed-loop system is thus stable.

B.3. Well posedness. We need to prove the well posedness of the loop $\Delta-M_{\Delta 1}$. As is well known (see Reference, 30), the interconnection of two causal operators is well posed if one of them is strictly causal. If $\Delta$ is strictly causal, then the closed-loop system is well posed. Assume now that $\Delta$ is not strictly causal. Let $D_{\Delta}$ be the memoryless part of $\Delta$. By loop shifting arguments, checking well posedness for the closed-loop system reduces to checking the well posedness of $D_{\Delta}-M_{\Delta 1}$.

Assume that this closed-loop system is not well posed, that is, there exists a $p \neq 0$ such that $\left(I-\Delta M_{\Delta 1}\right) p=0$. Let be $q=M_{\Delta 1} p$. Condition (35) implies that there exists a strictly positive $\varepsilon$ such that

$$
\left[\begin{array}{l}
q \\
p
\end{array}\right]^{\mathrm{T}}\left[\begin{array}{cc}
X S & Y S+G \\
Y^{\mathrm{T}} S+G & Z S
\end{array}\right]\left[\begin{array}{l}
q \\
p
\end{array}\right] \leqslant \varepsilon\left(q^{\mathrm{T}} q+p^{\mathrm{T}} p\right)
$$

But the class of considered $\Delta$ is such that $D_{\Delta}$ satisfies:

$$
\left[\begin{array}{l}
q \\
p
\end{array}\right]^{\mathrm{T}}\left[\begin{array}{cc}
X S & Y S+G \\
Y^{\mathrm{T}} S+G & Z S
\end{array}\right]\left[\begin{array}{l}
q \\
p
\end{array}\right] \geqslant 0
$$

So, if $p \neq 0$ and $q \neq 0$ then there is a contradiction between the quadratic constraints (38) and (39). The original closed-loop system is thus well posed.

\section{Control}

\section{Lemma C.1}

Let there be the matrix $D \in \mathbf{R}^{n \times n}$ and the full-rank matrices $D_{u} \in \mathbf{R}^{n \times k}, D_{y} \in \mathbf{R}^{l \times n}$ with $k \leqslant n$ and $l \leqslant n$. Let there be the matrices $X, Y, Z \in \mathbf{R}^{n \times n}$ such that $X=\operatorname{diag}\left(0, X_{2}\right), Y=\operatorname{diag}\left(Y_{1}, Y_{2}\right)$ and $Z=\operatorname{diag}\left(Z_{1}, Z_{2}\right)$. $X_{2}, Y_{2}, Z_{2} \in \mathbf{R}^{p \times p}$ with $p \leqslant n$.

Assume that $X_{2}>0$ and $Z_{2} \leqslant 0$ and $\left[\begin{array}{ll}X & Y \\ Y^{\mathrm{T}} & \frac{Y}{Z}\end{array}\right]$ is a full-rank matrix.

Then, there exists a matrix $X \in \mathbf{R}^{k \times l}$ such that

$$
\left[\begin{array}{c}
D+D_{u} K D_{y} \\
I_{n}
\end{array}\right]^{\mathrm{T}}\left[\begin{array}{cc}
X & Y \\
Y^{\mathrm{T}} & Z
\end{array}\right]\left[\begin{array}{c}
D+D_{u} K D_{y} \\
I_{n}
\end{array}\right]<0
$$

if and only if

$$
\begin{gathered}
D_{y}^{\perp^{\mathrm{T}}}\left[\begin{array}{c}
D \\
I_{n}
\end{array}\right]^{\mathrm{T}}\left[\begin{array}{cc}
X & Y \\
Y^{\mathrm{T}} & Z
\end{array}\right]\left[\begin{array}{c}
D \\
I_{n}
\end{array}\right] D_{y}^{\perp}<0 \\
D_{u}^{\mathrm{T} \perp^{\mathrm{T}}}\left[\begin{array}{c}
D^{\mathrm{T}} \\
I_{n}
\end{array}\right]^{\mathrm{T}}\left[\begin{array}{cc}
\tilde{X} & \tilde{Y} \\
\tilde{Y}^{\mathrm{T}} & \tilde{Z}
\end{array}\right]\left[\begin{array}{c}
D^{\mathrm{T}} \\
I_{n}
\end{array}\right] D_{y}^{\mathrm{T}^{\perp}}<0
\end{gathered}
$$

with

$$
\left[\begin{array}{cc}
X & Y \\
Y^{\mathrm{T}} & Z
\end{array}\right]\left[\begin{array}{cc}
-\tilde{Z} & \tilde{Y}^{\mathrm{T}} \\
\tilde{Y} & -\tilde{X}
\end{array}\right]=I
$$

Furthermore, if the conditions (41), (42), (43) hold then a possible $K$ satisfying (40) is given by:

$$
K=-\varepsilon^{-1} \mathscr{U} \Phi \mathscr{V}^{\mathrm{T}}\left(\mathscr{V} \Phi \mathscr{V}^{\mathrm{T}}\right)^{-1} \text { and } \Phi \triangleq\left(\varepsilon^{-1} \mathscr{U}^{\mathrm{T}} \mathscr{U}-\mathscr{G}\right)^{-1}
$$


with $\mathscr{G}, \mathscr{U}$ and $\mathscr{V}$ the matrices:

$$
\begin{aligned}
\mathscr{G} & =\left[\begin{array}{ccc}
D_{11}^{\mathrm{T}} Y_{1}+Y_{1}^{\mathrm{T}} D_{11}+Z_{1} & Y_{1}^{\mathrm{T}} D_{12} & D_{21}^{\mathrm{T}} \\
D_{12}^{\mathrm{T}} Y_{1} & Z_{2}-Y_{2}^{\mathrm{T}} X_{2}^{\mathrm{T}} Y_{2} & \left(D_{22}+X_{2}^{-1} Y_{2}\right)^{\mathrm{T}} \\
D_{21} & D_{22}+X_{2}^{-1} Y_{2} & -X_{2}^{-1}
\end{array}\right] \\
\mathscr{U} & =\left[\begin{array}{lll}
D_{u 1}^{\mathrm{T}} Y_{1} & 0 & D_{u 2}^{\mathrm{T}}
\end{array}\right] \\
\mathscr{V} & =\left[\begin{array}{ll}
D_{y} & 0
\end{array}\right] \\
D & =\left[\begin{array}{ll}
D_{11} & D_{12} \\
D_{21} & D_{22}
\end{array}\right]
\end{aligned}
$$

$\varepsilon$ is a positive scalar such that $\varepsilon \ll 1 / \mu$, with

$$
\mu=\max \left(0, \lambda_{\max }\left(\mathscr{U}^{+\mathrm{T}}\left(\mathscr{G}-\mathscr{G} \mathscr{U}^{\perp}\left(\mathscr{U}^{\perp \mathrm{T}} \mathscr{G} \mathscr{U}^{\perp}\right)^{-1} \mathscr{U}^{\perp \mathrm{T}} \mathscr{G}\right) \mathscr{U}^{+}\right)\right)
$$

Proof. First, we prove the equivalence between the inequality (40) and the inequalities (41) and (42).

Let there be $D=\left[\begin{array}{ll}D_{11} & D_{12} \\ D_{21} & D_{22}\end{array}\right]$ with $D_{22} \in \mathbf{R}^{p \times p}$ and $D_{u}=\left[\begin{array}{l}D_{u 1} \\ D_{u 2}\end{array}\right]$.

Using a square completion argument, the condition (40) is equivalent to:

$$
W^{\mathrm{T}} X_{2} W+V^{\mathrm{T}} Y_{1}\left[\begin{array}{ll}
I & 0
\end{array}\right]+\left[\begin{array}{l}
I \\
0
\end{array}\right] Y_{1}^{\mathrm{T}} V+\left[\begin{array}{cc}
Z_{1} & 0 \\
0 & Z_{2}-Y_{2}^{\mathrm{T}} X_{2} Y_{2}
\end{array}\right]<0
$$

with

$$
\begin{aligned}
W & =\left[\begin{array}{ll}
D_{21} & D_{22}
\end{array}\right]+D_{u 2} K D_{y}+X_{2}^{-1} Y_{2}\left[\begin{array}{ll}
0 & I
\end{array}\right] \\
V & =\left[\begin{array}{ll}
D_{11} & D_{12}
\end{array}\right]+D_{u 1} K D_{y}
\end{aligned}
$$

With $\tilde{X}_{2} \triangleq\left(Y_{2}^{\mathrm{T}} X_{2}^{-1} Y_{2}-Z_{2}\right)^{-1}$ and a Schur complement, it is equivalent to:

$$
\mathscr{G}+\mathscr{U}^{\mathrm{T}} K \mathscr{V}+\mathscr{V}^{\mathrm{T}} K^{\mathrm{T}} \mathscr{U}<0
$$

Applying the elimination lemma ${ }^{50,51}(45)$ is equivalent to the existence of a scalar $\sigma$ such that:

$$
\mathscr{G}<\sigma \mathscr{U}^{\mathrm{T}} \mathscr{U} \text { and } \mathscr{G}<\sigma \mathscr{V}^{\mathrm{T}} \mathscr{V}
$$

It is also equivalent to

$$
\mathscr{U}^{\perp \mathrm{T} \mathscr{G} \mathscr{U}} \mathscr{H}^{\perp}<0 \quad \text { and } \quad \mathscr{V}^{\perp \mathrm{T}} \mathscr{G} \mathscr{V}^{\perp}<0
$$

Applying the elimination lemma to our problem the following first condition is obtained:

$$
\left[\begin{array}{ccc}
D_{11}^{\mathrm{T}} Y_{1}+Y_{1}^{\mathrm{T}} D_{11}+Z_{1} & Y_{1}^{\mathrm{T}} D_{12} & D_{21}^{\mathrm{T}} \\
D_{12}^{\mathrm{T}} Y_{1} & -X_{2}^{-1} & \left(D_{22}+X_{2}^{-1} Y_{2}\right)^{\mathrm{T}} \\
D_{21} & D_{22}+X_{2}^{-1} Y_{2} & -X_{2}^{-1}
\end{array}\right]<\sigma\left[\begin{array}{c}
Y_{1}^{\mathrm{T}} D_{u 1} \\
0 \\
D_{u 2}
\end{array}\right]\left[\begin{array}{lll}
D_{u 1}^{\mathrm{T}} Y_{1} & 0 & D_{u 2}^{\mathrm{T}}
\end{array}\right]
$$

which is equivalent to

$$
\left[\begin{array}{ccc}
Y_{1}^{-\mathrm{T}} D_{11}^{\mathrm{T}}+D_{11} Y_{1}^{-1}+Y_{1}^{-\mathrm{T}} Z_{1} Y_{1}^{-1} & Y_{1}^{-\mathrm{T}} D_{21}^{\mathrm{T}} & D_{12} \\
D_{21} Y_{1}^{-\mathrm{T}} & -X_{2}^{-1} & D_{22}+X_{2}^{-1} Y_{2} \\
D_{12}^{\mathrm{T}} & \left(D_{22}+X_{2}^{-1} Y_{2}\right)^{\mathrm{T}} & -X_{2}^{-1}
\end{array}\right]<\sigma\left[\begin{array}{c}
D_{u} \\
0
\end{array}\right]\left[\begin{array}{ll}
D_{u}^{\mathrm{T}} & 0
\end{array}\right]
$$

$Y_{1}$ is invertible because the matrix $\left[\begin{array}{cc}0 & Y_{1}^{\mathrm{T}} \\ Y_{1}^{\mathrm{T}} & \mathrm{Z}_{1}^{1}\end{array}\right]$ is, by assumption, full rank. 
With $X_{2}^{-1} Y_{2}=\tilde{Y}_{2}^{\mathrm{T}} \tilde{X}_{2}^{-1}$, a Schur complement and $\tilde{Z}_{2}=Y_{2}^{\mathrm{T}} \tilde{X}_{2}^{-1} Y_{2}-X_{2}^{-1}, \tilde{Y}_{1}=Y_{1}^{-1}, \tilde{Z}_{1}=Y_{1}^{-\mathrm{T}} Z_{1} Y_{1}^{-1}$, we obtain the following condition:

$$
\left[\begin{array}{l}
D^{\mathrm{T}} \\
I_{n}
\end{array}\right]^{\mathrm{T}}\left[\begin{array}{cc}
\tilde{X} & \tilde{Y} \\
\tilde{Y}^{\mathrm{T}} & \tilde{Z}
\end{array}\right]\left[\begin{array}{c}
D^{\mathrm{T}} \\
I_{n}
\end{array}\right]<\sigma D_{u} D_{u}^{\mathrm{T}}
$$

with $\tilde{X}=\operatorname{diag}\left(0, \tilde{X}_{2}\right), \tilde{Y}=\operatorname{diag}\left(\tilde{Y}_{1}, \tilde{Y}_{2}\right)$ and $\tilde{Z}=\operatorname{diag}\left(\tilde{Z}_{1}, \tilde{Z}_{2}\right)$. This condition is then equivalent to (42).

Consider the second condition obtained by the application of the elimination lemma

$$
\left[\begin{array}{ccc}
D_{11}^{\mathrm{T}} Y_{1}+Y_{1}^{\mathrm{T}} D_{11}+Z_{1} & Y_{1}^{\mathrm{T}} D_{12} & D_{21}^{\mathrm{T}} \\
D_{12}^{\mathrm{T}} Y_{1} & -X_{2}^{-1} & \left(D_{22}+X_{2}^{-1} Y_{2}\right)^{\mathrm{T}} \\
D_{21} & D_{22}+X_{2}^{-1} Y_{2} & -X_{2}^{-1}
\end{array}\right]<\sigma\left[\begin{array}{c}
D_{y}^{\mathrm{T}} \\
0
\end{array}\right]\left[\begin{array}{ll}
D_{y} & 0
\end{array}\right]
$$

With a Schur complement, we obtain the following condition:

$$
\left[\begin{array}{l}
D \\
I_{n}
\end{array}\right]^{\mathrm{T}}\left[\begin{array}{cc}
X & Y \\
Y^{\mathrm{T}} & Z
\end{array}\right]\left[\begin{array}{c}
D \\
I_{n}
\end{array}\right]<\sigma D_{y}^{\mathrm{T}} D_{y}
$$

This condition is then equivalent to (41).

A possible $K$ can be obtained by a direct adaptation of Theorem 1 of the paper. ${ }^{52}$ We just need to point out that:

- as $D_{u}$ and $D_{y}$ are full-rank matrices, $\mathscr{U}$ and $\mathscr{V}$ are full-rank matrices too.

- $\varepsilon>0$ must be chosen small enough such that if (45) holds then

$$
\mathscr{G}+\mathscr{U}^{\mathrm{T}} K \mathscr{V}+\mathscr{V}^{\mathrm{T}} K^{\mathrm{T}} \mathscr{U}+\varepsilon^{\mathrm{T}} K^{\mathrm{T}} K \mathscr{V}<0
$$

By the Schur complement formula, it is equivalent to:

$$
\left[\begin{array}{cc}
\mathscr{G}+\mathscr{U}^{\mathrm{T}} K \mathscr{V}+\mathscr{V}^{\mathrm{T}} K^{\mathrm{T}} \mathscr{U} & \mathscr{V}^{\mathrm{T}} K^{\mathrm{T}} \\
K \mathscr{V} & -\varepsilon^{-1} I
\end{array}\right]<0
$$

Applying the elimination lemma, this LMI is equivalent to the existence of a $\sigma$ such that:

$$
\begin{aligned}
\mathscr{G} & <\sigma^{\mathscr{V}^{\mathrm{T}} \mathscr{V}} \\
\varepsilon \sigma & >-1 \\
\mathscr{G} & <\frac{\sigma}{1+\varepsilon \sigma} \mathscr{U}^{\mathrm{T}} \mathscr{U}
\end{aligned}
$$

Applying Lemma 3 of the paper, ${ }^{53} \sigma$ and $\varepsilon$ are chosen such that:

$$
\begin{aligned}
\sigma & >\lambda_{\text {max }}\left(\mathscr{V}^{+\mathrm{T}}\left(\mathscr{G}-\mathscr{G} \mathscr{V}^{\perp}\left(\mathscr{V}^{\perp \mathrm{T}} \mathscr{G} \mathscr{V}^{\perp}\right)^{-1} \mathscr{V}^{\perp \mathrm{T}} \mathscr{G}\right) \mathscr{V}^{+}\right) \\
\varepsilon \sigma & >-1 \\
\sigma & >\lambda_{\max }\left(\mathscr{U}^{+\mathrm{T}}\left(\mathscr{G}-\mathscr{G} \mathscr{U}^{\perp}\left(\mathscr{U}^{\perp \mathrm{T}} \mathscr{G} \mathscr{U}^{\perp}\right)^{-1} \mathscr{U}^{\perp \mathrm{T}} \mathscr{G}\right) \mathscr{U}^{+}\right)(1+\varepsilon \sigma)
\end{aligned}
$$

For instance, take $\sigma \rightarrow+\infty$ and $\varepsilon \ll 1 / \lambda_{\max }\left(\mathscr{U}^{+\mathrm{T}}\left(\mathscr{G}-\mathscr{G} \mathscr{U}^{\perp}\left(\mathscr{U}^{\perp \mathrm{T}} \mathscr{G} \mathscr{U}^{\perp}\right)^{-1} \mathscr{U}^{\perp \mathrm{T}} \mathscr{G}\right) \mathscr{U}^{+}\right)$.

This achieves the proof.

This lemma is now applied to prove Theorem 3.1. Without any loss of generality and to avoid cumbersome notation, we assume that $\Delta$ has only one block of dimension $n_{r} \times n_{r}$. A robust gain-scheduled controller $u=\mathscr{F}_{u}\left(K, \Delta_{K}\right)_{y}$ is sought such that, connected to the system:

$$
\left[\begin{array}{l}
z(t) \\
y(t)
\end{array}\right]=\mathscr{F}_{u}\left(\left[\begin{array}{ccc}
M_{q p} & M_{q w} & M_{q u} \\
M_{z p} & M_{z w} & M_{z u} \\
M_{y p} & M_{y w} & 0
\end{array}\right], \Delta\right)\left[\begin{array}{c}
w(t) \\
u(t)
\end{array}\right]
$$


it ensures, for the closed-loop system, (i) stability and (ii) $\left\{X_{\text {perf }}, Y_{\text {perf }}, Z_{\text {perf }}\right\}$-dissipativity between $w$ and $z$. The equations of the closed-loop system are: $z=\mathscr{F}_{u}\left(\bar{M}, \operatorname{diag}\left(\Delta, \Delta_{K}\right)\right) w$ with

$$
\bar{M}=\left[\begin{array}{ccc}
M_{q p} & 0 & M_{q w} \\
0 & 0 & 0 \\
M_{z p} & 0 & M_{z w}
\end{array}\right]+\left[\begin{array}{cc}
0 & M_{q u} \\
I & 0 \\
0 & M_{z u}
\end{array}\right] K\left[\begin{array}{ccc}
0 & I & 0 \\
M_{y p} & 0 & M_{y w}
\end{array}\right]
$$

Depending on the class of $\Delta, \Delta_{K}$ can have different structures:

- $\Delta$ belongs to $\mathscr{C}_{1}$ or $\mathscr{C}_{3}$ (that is, $\Delta=\delta I_{n}$ ): $\Delta_{K}=\delta I_{k}$ wih $k<n$,

- $\Delta$ belongs to $\mathscr{C}_{5}$ or $\mathscr{C}_{6}: \Delta_{K}=I_{0}$,

- $\Delta$ belongs to $\mathscr{C}_{2}$ or $\mathscr{C}_{4}: \Delta_{K}=\Delta$ or $\Delta_{K}=I_{0}$.

Define $k$ as the size of $\Delta_{k}$. The operator $\operatorname{diag}\left(\Delta, \Delta_{K}\right)$ is then $\{\bar{X}, \bar{Y}, \bar{Z}\}$-dissipative with $\bar{X}=\operatorname{diag}\left(X, X^{k}\right)$, $Y=\operatorname{diag}\left(Y, Y^{k}\right), \bar{Z}=\operatorname{diag}\left(Z, Z^{k}\right)$ and with the corresponding scalings

$$
\bar{S}=\left[\begin{array}{ll}
S & * \\
* & *
\end{array}\right] \quad \bar{G}=\left[\begin{array}{ll}
G & * \\
* & *
\end{array}\right]
$$

where $S \in \mathscr{S}(n)$ and $G \in \mathscr{G}(n)$. By application of Lemma 3.2, the closed-loop system is stable and $\left\{X_{\text {per } f}, Y_{\text {per } f}, Z_{\text {per } f}\right\}$-dissipative if

$$
\left[\begin{array}{c}
\bar{M} \\
I_{\left(n+n_{w}\right)}
\end{array}\right]^{\mathrm{T}} \mathscr{M}\left[\begin{array}{c}
\bar{M} \\
I_{\left(n+n_{w}\right)}
\end{array}\right]<0
$$

where

$$
\mathscr{M}=\mathscr{P}_{M}^{\mathrm{T}} \operatorname{diag}\left(\left[\begin{array}{cc}
\bar{X} \bar{S} & \bar{Y} \bar{S}+\bar{G} \\
\bar{Y}^{\mathrm{T}} \bar{S}+\bar{G}^{\mathrm{T}} & \bar{Z} \bar{S}
\end{array}\right],-\left[\begin{array}{cc}
Z_{\text {perf }} & Y_{\text {perf }}^{\mathrm{T}} \\
Y_{\text {perf }} & X_{\text {perf }}
\end{array}\right]\right) \mathscr{P}_{M}
$$

Lemma C.1 is then applied. Condition (43) leads to the two relations

$$
\begin{gathered}
{\left[\begin{array}{cc}
\bar{X} \bar{S} & \bar{Y} \bar{S}+\bar{G} \\
\bar{Y}^{\mathrm{T}} \bar{S} & \bar{Z} \bar{S}
\end{array}\right]\left[\begin{array}{cc}
-\tilde{\bar{Z}} \bar{T} & \tilde{\bar{Y}} \bar{T}+\bar{H}^{\mathrm{T}} \\
\tilde{\bar{Y}} \bar{T}+\bar{H} & -\tilde{\tilde{X}} \bar{T}
\end{array}\right]=I} \\
{\left[\begin{array}{cc}
Z_{\text {perf }} & Y_{\text {perf }} \\
Y_{\text {perf }}^{\mathrm{T}} & Z_{\text {perf }}
\end{array}\right]\left[\begin{array}{cc}
-\tilde{Z}_{\text {perf }} & \tilde{Y}_{\text {perf }}^{\mathrm{T}} \\
\tilde{Y}_{\text {perf }} & -\tilde{X}_{\text {perf }}
\end{array}\right]=I}
\end{gathered}
$$

Consider the first equality of (47). Let us split $\bar{T}$ and $\bar{H}$ in the following way:

$$
\bar{T}=\left[\begin{array}{ll}
T & * \\
* & *
\end{array}\right] \quad \bar{H}=\left[\begin{array}{cc}
H & * \\
* & *
\end{array}\right]
$$

where $T \in \mathscr{S}(n)$ and $H \in \mathscr{G}(n)$.

First, assume that $\bar{G}=0$ ( $\Delta$ does not belong to the class $\left.\mathscr{C}_{3}\right)$. As $\overline{\mathscr{S}}$ commutes with $\bar{X}, \bar{Y}, \bar{Z}$, the first equality of (47) is implied by the following equalities:

$$
\begin{aligned}
{\left[\begin{array}{cc}
X & Y \\
Y^{\mathrm{T}} & Z
\end{array}\right]\left[\begin{array}{cc}
-\tilde{Z} & \tilde{Y}^{\mathrm{T}} \\
\tilde{Y} & -\tilde{X}
\end{array}\right] } & =I \\
\bar{S} \bar{T} & =I
\end{aligned}
$$

Then, using the completion Lemma 6.2 proposed by Packard in Reference 4, $\bar{S} \bar{T}=I$ and $\bar{S}, \bar{T}>0$ if and only if

$$
\left[\begin{array}{cc}
S & I \\
I & T
\end{array}\right]>0 \text { and } \operatorname{rank}\left[\begin{array}{cc}
S & I \\
I & T
\end{array}\right] \leqslant n+k
$$


For all the classes, except $\mathscr{C}_{3}$, the constraints of Table IV (Section 3) are obtained. Furthermore, the proof of this completion lemma suggests a method to obtained $\bar{S}$ from $S$ and $T$ :

$$
\bar{S}=\left[\begin{array}{cc}
S & R \\
R^{\mathrm{T}} & I
\end{array}\right]
$$

with $R$ such that: $S-T^{-1}=R R^{\mathrm{T}}$.

Now, assume that $\Delta \in \mathscr{C}_{3}$. In this case $X=x I, Y=y I$ and $Z=z I$. $\tilde{X}, \tilde{Y}$ and $\tilde{Z}$ are chosen such that:

$$
\left[\begin{array}{cc}
X & Y \\
Y^{\mathrm{T}} & Z
\end{array}\right]\left[\begin{array}{cc}
-\tilde{Z} & \tilde{Y}^{\mathrm{T}} \\
\tilde{Y} & -\tilde{X}
\end{array}\right]=I
$$

Then, introducing $\gamma=y^{2}-x z$, the condition (47) is equivalent to:

$$
\begin{aligned}
\bar{S} \bar{T}+y\left(\gamma^{-1} \bar{G} \bar{T}+\bar{S} \bar{H}\right)+\bar{G} \bar{H} & =I \\
\bar{G}^{\mathrm{T}} \bar{T} & =\gamma \bar{S} \bar{H} \\
\bar{S} \bar{T}+y\left(\bar{S} \bar{H}^{\mathrm{T}}+\gamma^{-1} \bar{G} \bar{T}\right)+\bar{G}^{\mathrm{T}} \bar{H}^{\mathrm{T}} & =I
\end{aligned}
$$

Noting that $\bar{H}$ and $\bar{G}$ are skew-symmetric matrices, this is equivalent to the condition:

$$
\left(\bar{S}+\Gamma^{-1} \bar{G}\right)(\bar{T}+\Gamma \bar{H})=I
$$

with $\Gamma=\gamma^{1 / 2} I$.

Using the completion lemma proposed by Helmersson in the paper, ${ }^{10}\left(\bar{S}+\Gamma^{-1} \bar{G}\right)(\bar{T}+\Gamma \bar{H})=I$ and $\bar{S}, \bar{T}>0$ if and only if $S, T>0$ and

$$
\operatorname{rank}\left[\begin{array}{cc}
S+\Gamma^{-1} G & I \\
I & T+\Gamma H
\end{array}\right]=n+k
$$

Furthermore,

$$
\bar{S}=\left[\begin{array}{cc}
S & R \\
R^{\mathrm{T}} & U
\end{array}\right] \quad \bar{G}=\left[\begin{array}{cc}
G & Q \\
-Q^{\mathrm{T}} & V
\end{array}\right]
$$

with $S+\Gamma^{-1} G-(T+\Gamma H)^{-1}=N M$ where $N \in \mathbf{R}^{n \times k}$ and $M \in \mathbf{R}^{k \times n}$.

$$
\left[\begin{array}{cc}
S & R \\
R^{\mathrm{T}} & U
\end{array}\right]+\left[\begin{array}{cc}
\Gamma^{-1} & 0 \\
0 & \Gamma^{k^{-1}}
\end{array}\right]\left[\begin{array}{cc}
G & Q \\
-Q^{\mathrm{T}} & V
\end{array}\right]=\left[\begin{array}{ccc}
S+\Gamma^{-1} G & N B & 0 \\
M & B & 0 \\
0 & 0 & I
\end{array}\right]
$$

with $B=2\left(N^{\mathrm{T}} S^{-1} N\right)^{-1}\left(I-\frac{1}{2} N^{\mathrm{T}} S^{-1} M^{\mathrm{T}}\right)$.

After some manipulations, the conditions (41) and (42) reduce to the conditions (12) and (13). Note that only $T$ and $S$ are in these two last conditions.

Explicit expression for the controller. Now we consider the general case, that is $\Delta=\operatorname{diag}\left(\Delta_{i}\right)$. We assume that the existence conditions of Theorem 3.1 hold. The purpose of this section is to propose a possible expression for the matrix $K$ of the robust gain-scheduled controller.

We shall derive the proof for the case when $X_{1}=0$ (to cover the case when $\Delta$ contains an integrator) and for the other blocks in $\Delta, i \in[2, r], X_{i}>0$ (recall $r$ is the number of blocks in $\Delta$ ).

Define

$$
k_{i}=\operatorname{rank}\left[\begin{array}{cc}
S_{i}+\Gamma_{i}^{-1} G_{i} & I \\
I & T_{i}+\Gamma_{i} H_{i}
\end{array}\right]-n_{i}
$$


The expression (44) of Lemma C.1 is than applied with:

$$
\begin{gathered}
Y_{1}=\operatorname{diag}\left(Y_{1}, Y_{1}^{k_{1}}\right)\left[\begin{array}{ll}
S_{1} & R_{1} \\
R_{1}^{\mathrm{T}} & U_{1}
\end{array}\right]+\left[\begin{array}{ll}
G_{1} & Q_{1} \\
Q_{1}^{\mathrm{T}} & V_{1}
\end{array}\right] \\
Z_{1}=\operatorname{diag}\left(Z_{1}, Z_{1}^{k_{1}}\right)\left[\begin{array}{ll}
S_{1} & R_{1} \\
R_{1}^{\mathrm{T}} & U_{1}
\end{array}\right] \\
X_{2}=\operatorname{diag}\left(\operatorname{diag}\left(\operatorname{diag}\left(X_{i}\right), \operatorname{diag}\left(X_{1}^{k_{i}}\right)\right)\left[\begin{array}{ll}
\operatorname{diag}\left(S_{i}\right) & \operatorname{diag}\left(R_{i}\right) \\
\operatorname{diag}\left(R_{i}^{\mathrm{T}}\right) & \operatorname{diag}\left(U_{i}\right)
\end{array}\right],-Z_{\text {perf }}\right) \\
Y_{2}=\operatorname{diag}\left(\operatorname{diag}\left(\operatorname{diag}\left(Y_{i}\right), \operatorname{diag}\left(Y_{1}^{k_{i}}\right)\right)\left[\begin{array}{ll}
\operatorname{diag}\left(S_{i}\right) & \operatorname{diag}\left(R_{i}\right) \\
\operatorname{diag}\left(R_{i}^{\mathrm{T}}\right) & \operatorname{diag}\left(U_{i}\right)
\end{array}\right]+\left[\begin{array}{ll}
\operatorname{diag}\left(G_{i}\right) & \operatorname{diag}\left(Q_{i}\right) \\
\operatorname{diag}\left(Q_{i}\right) & \operatorname{diag}\left(V_{i}\right)
\end{array}\right],-Y_{\text {perf }}^{\mathrm{T}}\right) \\
Z_{2}=\operatorname{diag}\left(\operatorname{diag}\left(\operatorname{diag}\left(Z_{i}\right), \operatorname{diag}\left(Z_{1}^{\left.k_{i}\right)}\right)\left[\begin{array}{ll}
\operatorname{diag}\left(S_{i}\right) & \operatorname{diag}\left(R_{i}\right) \\
\operatorname{diag}\left(R_{i}^{\mathrm{T}}\right) & \operatorname{diag}\left(U_{i}\right)
\end{array}\right],-X_{\text {perf }}\right)\right.
\end{gathered}
$$

As it was previously explained, $Q_{i}, R_{i}, U_{i}$ and $V_{i}$ are obtained using the completion lemmas of Packard and Helmersson. The matrices $M, M_{u}$ and $M_{y}$ are split in the following way:

$$
\left[\begin{array}{c|c}
M & M_{u} \\
\hline M_{y} & 0
\end{array}\right]=\left[\begin{array}{ccc|c}
M_{q p 11} & M_{q p 12} & M_{q w 1} & M_{q u 1} \\
M_{q p 21} & M_{q p 22} & M_{q w 2} & M_{q u 2} \\
M_{z p 1} & M_{z p 2} & M_{z w} & M_{z u} \\
\hline M_{z p 1} & M_{y p 2} & M_{y w} & 0
\end{array}\right]
$$

where $M_{q p 11}$ has the same size as $X_{1}$. Then, take:

$$
\begin{aligned}
& {\left[\begin{array}{c|c|c}
D_{11} & D_{12} & D_{u 1} \\
\hline D_{21} & D_{22} & D_{u 2}
\end{array}\right]=\left[\begin{array}{cc|ccc|ccc}
M_{q p 11} & 0 & M_{q p 12} & 0 & M_{q w 1} & 0 & 0 & M_{q u 1} \\
0 & 0 & 0 & 0 & 0 & I & 0 & 0 \\
\hline M_{q p 21} & 0 & M_{q p 22} & 0 & M_{q w 2} & 0 & 0 & M_{q u 2} \\
0 & 0 & 0 & 0 & 0 & 0 & I & 0 \\
M_{z p 1} & 0 & M_{z p 2} & 0 & M_{z w} & 0 & 0 & M_{z u}
\end{array}\right]} \\
& D_{y}=\left[\begin{array}{ccccc}
0 & {\left[\begin{array}{l}
I \\
0
\end{array}\right]} & 0 & {\left[\begin{array}{l}
0 \\
I
\end{array}\right]} & 0 \\
M_{y p 1} & 0 & M_{y p 2} & 0 & M_{y w}
\end{array}\right]
\end{aligned}
$$

The expression (44) of Lemma C.1 is applied with the above matrices. This concludes our proof.

\section{ACKNOWLEDGEMENTS}

The authors wish to thank the reviewers for valuable comments and help that led to improve the first version of this paper. The first author wishes to thank Vin cent Fromion and Stéphane Font from the Service Automatique, ESE, Paris for valuable discussions and Gilles Duc, also from ESE, for support. He also wishes to thank one of the reviewers of the conference paper ${ }^{28}$ for useful comments. 


\section{REFERENCES}

1. Doyle, J., A. Packard and K. Zhou, 'Review of LFT's, LMI's and $\mu$ ', Proc. IEEE Conf. on Decision and Control, volume 2, Brighton, UK, December 1991, pp. 1227-1232.

2. Packard, A., K. Zhou, P. Pandey and G. Becker, 'A collection of robust control problems leading to LMI's', Proc. IEEE Conf. on Decision and Control, volume 2, Brighton, UK. December 1991, pp. 1245-1250.

3. Lu, W. M., K. Zhou and J. C. Doyle, 'Stabilization of LFT systems', Proc. IEEE Conf. on Decision and Control, Brighton, UK, December 1991, pp. 1238-1244.

4. Packard, A., 'Gain scheduling via linear fractional transformations', Syst. Control Letters, 22(2), 79-92 (1994).

5. Apkarian, P. and P. Gahinet, 'A convex characterization of gain-scheduled $H_{\infty}$ controllers', IEEE Trans. Automat Control, 40(5), 853-864 (1995).

6. Packard, A., G. Becker, D. Philbrick and G. Balas, 'Control of parameter-dependent systems: applications to $H_{\infty}$ gain-scheduling', Proc. American Control Conf., San Francisco, CA, May 1993, pp 328-333.

7. Becker, G., A. Packard, D. Philbrick and G. Balas, 'Control of parametrically dependent linear systems: a single quadratic Lyapunov approach', 1993 American Control Conference, volume 3, June 1993, pp. 2795-2799.

8. Apkarian, P., P. Gahinet and G. Becker, 'Self-scheduled $H_{\infty}$ control of linear parameter-varying systems', Proc. American Control Conf., Baltimore, Maryland, June 1994, pp. 856-860.

9. Becker, G. and A. Packard, 'Robust performance of linear parametrically varying systems using parametricallydependent linear feedback', Syst. Control Letters, 23(3), 205-215 (1994).

10. Helmersson, A., ' $\mu$ synthesis and LFT gain scheduling with mixed uncertainties', Proc. European Control Conf., Roma, Italy, 1995, pp. 153-158.

11. Helmersson, A., 'Methods for robust gain scheduling', Ph.D. Thesis, Linköping University, November 1995.

12. Wu, F., X. H. Yang, A. Packard and G. Becker, 'Induced $\mathscr{L}_{2}$ norm control for LPV system with bounded parameter variation rates', Proc. American Control Conf., Seattle, Washington, June 1995, pp. 2379-2383.

13. Fromion, V., S. Monaco and D. Normand-Cyrot, 'Robustness and stability of LPV plants through frozen system analysis', Int. J. Robust and Nonlinear Control, 6, 235-248 (1996).

14. Willems, J. C., 'Qualitative behavior of interconnected systems', Annals of Systems Research, 3, 61-80 (1973).

15. Sandell, N. R., P. Varaiya, M. Athans and M. G. Safonov, 'Survey of decentralized control methods for large scale systems', IEEE Trans. Automat Control, 23, 108-128 (1978).

16. Hill, D.J. and P.J. Moylan, 'Dissipative dynamical systems: basic input-output and state properties', J. Franklin Inst., 309(5), 327-357 (1980).

17. Safonov, M. G., Stability and Robustness of Multivariable Feedback Systems, MIT Press, Cambridge, 1980.

18. Rantzer, A. and A. Megretski, 'System analysis via integral quadratic constraints', Proc. IEEE Conf. on Decision and Control, Lake Buena Vista, Florida, December 1994, pp. 3062-3067.

19. Glattfelder, A.H. and W. Schaufelberger, 'Stability analysis of single loop control systems with saturation and anti reset-windup circuits', IEEE Trans. Automat Control, 28(12), 1074-1081 (1983).

20. Doyle, J. C., R. S. Smith and D. F. Enns, 'Control of plants with input saturation nonlinearities', Proc. IEEE Conf. on Decision and Control, Minneapolis, MN, 1987, pp. 1034-1038.

21. Kothare, M. V., P. J. Campo and M. Morari, 'A unified framework for the study of anti-windup reset', Technical Report CIT-CDS 93-011, Caltech, June 1993.

22. Bernstein, D. S. and W. M. Haddad, 'Nonlinear controllers for positive-real systems with arbitrary input nonlinearities', Proc. American Control Conf., volume 1, San Francisco, California, June 1993, pp. 832-836.

23. Kavranoğlu, D. and M. Bettayeb, 'Characterization of the solution to the optimal $H_{\infty}$ model reduction problem', Syst. Control Letters, 20(2), 99-107 (1993).

24. Wang, W., J. Doyle and K. Glover, 'Model reduction of LFT systems', Proc. IEEE Conf. on Decision and Control, volume 2, Brighton, UK, December 1991, pp. 1233-1238.

25. Beck, C., J. C. Doyle and K. Glover, 'Model reduction of multi-dimensional and uncertain systems', Technical Report CIT/CDS 94-017, California Institut of Technology, November 1994.

26. Beck, C., J. C. Doyle and K. Glover, 'Model reduction of multi-dimensional and uncertain systems', IEEE Trans. Automat Control, 41(10), 1466-1477 (1996).

27. El Ghaoui, L. and G. Scorletti, 'Control of rational systems using linear-fractional representations and linear matrix inequalities', Automatica, 32(9), 1273-1284 (1996).

28. Scorletti, G. and L. El Ghaoui, 'Improved linear matrix inequalities conditions for gain-scheduling', Proc. IEEE Conf. on Decision and Control, New Orleans, Louisiana, December 1995, pp. 3626-3631.

29. Scorletti, G., 'Some results about the stabilization and the $\mathscr{L}_{2}$-gain control of LFT systems', Proc. IEEE IMACS Multiconference on Comp. Eng. in Syst. Appl., Lilles, France, July 1996, pp. 1240-1245.

30. Willems, J. C., The Analysis of Feedback Systems, volume 62 of Research Monographs, MIT Press, Cambridge, Massachusetts, 1969.

31. Zhou, K., J. C. Doyle and K. Glover, Robust and Optimal Control, Prentice Hall, New Jersey, 1995.

32. Vidyasagar, M., Input/Output Analysis of Large-scale Interconnected Systems, Number 29 in Lecture Notes in Control and Information Sciences, Springer-Verlag, Berlin, 1981. 
33. Goh, K.C. and M.G. Safonov, 'Robust analysis, sectors and quadratic functionals', Proc. IEEE Conf. on Decision and Control, New Orleans, Louisiana, USA, 1995, pp. 1988-1993.

34. Vandenberghe, L. and S. Boyd, 'Semidefinite programming', SIAM Review, 38(1), 49-95 (1996).

35. Nesterov, Y. and A. Nemirovsky, Interior-point Polynomial Methods in Convex Programming, Number 13 in Studies in Applied Mathematics, SIAM, Philadelphia, 1994.

36. Boyd, S. and L. El Ghaoui, 'Method of centers for minimizing generalized eigenvalues', Linear Algebra and Applications, special issue on Numerical Linear Algebra Methods in Control, Signals and Systems, 188, 1171-1176 (1993).

37. El Ghaoui, L., F. Oustry and M. Aitrami, 'A cone complementary linearization algorithm for static output-feedback and related problems', IEEE Trans. Automat Control, 42(8), (1997).

38. Goh, K. C., L. Turan, M. G. Safonov, G. P. Papavassilopoulos and J. H. Ly, 'Biaffine matrix inequality properties and computational methods', Proc. American Control Conf., Baltimore, Maryland, June 1994, pp. 850-851.

39. Pyatnitskii, E. S., 'Absolute stability of nonstationary nonlinear systems', Automation and Remote Control, 31(1), 1-11 (1970).

40. Wood, G. D., P. J. Goddard and K. Glover, 'Approximation of linear parameter-varying systems', Proc. IEEE Conf. on Decision and Control, December 1996.

41. Doyle, J. C., 'Analysis of feedback systems with structured uncertainties', IEE Proc., 129-D(6), 242-250 (1982).

42. Sideris, A., 'Elimination of frequency search for robustness tests', IEEE Trans. Automat Control, 37(10), 1635-1640 (1992).

43. El Ghaoui, L. and H. Lebret, 'Robust solutions to least-squares problems with uncertain data matrices', SIAM J. Matrix Anal. Appl., (1996) to appear.

44. Smith, R. and A. Packard, 'Optimal control of perturbed linear static systems', IEEE Trans. Automat Control, 41(4), 579-584 (1996).

45. El Ghaoui, L., R. Nikoukhah and F. Delebecque, LMITOOL: A Front-end for LMI Optimization, Users's Guide, February 1995. Available via anonymous ftp to ftp.ensta.fr, under /pub/elghaoui/Imitool.

46. Megretsky, A. and S. Treil, 'Power distribution inequalities in optimization and robustness of uncertain systems', Journal of Mathematical Systems, Estimation, and Control, 3(3), 301-319 (1993).

47. Shamma, J. S., 'Robust stability with time-varying systems structured uncertainty', IEEE Trans. Automat Control, 39(4), 714-724, (1994).

48. El Ghaoui, L. and J. P. Folcher, 'Multiobjective robust control of LTI systems subject to unstructured perturbations', Syst. Control Letters, 28, 23-30 (1996).

49. Yakubovich, V. A., 'Minimization of quadratic functionals under the quadratic constraints and the necessity of a frequency condition in the quadratic criterion for absolute stability of nonlinear control systems', Soviet Math. Doklady, 14(2), 593-597 (1973).

50. Boyd, S., L. El Ghaoui, E. Feron and V. Balakrishnan, Linear Matrix Inequalities in Systems and Control Theory, volume 15 of Studies in Appl. Math. SIAM, Philadelphia, June 1994.

51. Gahinet, P. and P. Apkarian, 'A linear matrix inequality approach to $H_{\infty}$ control', Int. J. Robust and Nonlinear Control, 4, 421-448 (1994)

52. Iwasaki, T. and R. E. Skelton, 'All controllers for the general $H_{\infty}$ control problem: LMI existence conditions and state space formulas', Automatica, 30(8), 1307-1317 (1994). 